INRA Prod. Anim., 2010, 23 (1), 65-80

\section{Robustesse, flexibilité, plasticité : des processus adaptatifs révélés dans les systèmes d'élevage extensifs de ruminants}

\author{
F. BLANC 1,2, B. DUMONT 3 , G. BRUNSCHWIG ${ }^{1,2}$, F. BOCQUIER ${ }^{4,5,6}$, J. AGABRIEL 3
}

${ }^{1}$ Clermont Université, VetAgroSup, UR EPR 2008.03.102, MC Sciences animales, BP 10448,

Site de Marmilhat, F-63370 Lempdes

2 VetAgroSup, Campus agronomique de Clermont, USC INRA 2005, Site de Marmilhat, F-63370 Lempdes

${ }^{3}$ INRA, UR1213 Recherche Herbivores, F-63122 Saint-Genès-Champanelle, France

${ }^{4}$ INRA, UMR868 Élevage des Ruminants en Régions Chaudes, 2 place Viala, F-34060 Montpellier, France

5 Montpellier SupAgro, Élevage des Ruminants en Régions Chaudes, F-34060 Montpellier, France

${ }^{6}$ CIRAD, Élevage des Ruminants en Régions Chaudes, F-34060 Montpellier, France

Courriel :f.blanc@vetagro-sup.fr

Les systèmes d'élevage extensifs de ruminants doivent assurer une double finalité : produire et entretenir les milieux. La pérennité de tels systèmes est conditionnée par l'aptitude des animaux à s'adapter tout en maintenant leur capacité à produire et à se reproduire. Dans un environnement très variable en termes de ressource alimentaire, peu contrôlé et soumis aux aléas, la variabilité individuelle des réponses adaptatives est perçue comme un élément générateur de la robustesse des animaux et de la productivité du troupeau.

Face à un environnement physique, climatique, économique et/ou politique changeant et incertain, les systèmes d'élevage doivent être capables de s'adapter pour durer (Dedieu et al 2008, Mosnier 2009). Cette capacité des systèmes à s'adapter repose en partie sur les potentiels adaptatifs des éléments qui les composent et notamment, sur ceux des animaux (Blanc et al 2006).

La notion d'adaptation désigne à la fois un état (adéquation de l'organisme à son milieu, viabilité) et un processus (transformations s'opérant au niveau de l'individu ou de l'espèce qui permettent d'atteindre cette adéquation). Le point de vue que nous privilégions dans ce texte est celui discuté par Alcaras et Lacroux (1999) dans le cadre de la systémique et qui met l'accent sur la capacité qu'ont les systèmes à se déformer pour répondre aux contraintes ou aléas de l'environnement. Dans cette approche, l'adaptation est perçue comme un processus qui conduit à la mise en œuvre d'une diversité de formes d'organisation permettant au système de résister (système rigide), de se déformer (système élastique ou flexible) ou de se transformer (système plastique) afin de répondre à des perturbations de l'environnement (figure 1).
Cette définition de l'adaptation renvoie à deux attitudes possibles quant à la façon d'aborder l'étude des processus adaptatifs. L'une consiste à s'intéresser aux mécanismes adaptatifs et à leurs déterminismes : comment se construit la réponse adaptative ? La seconde porte davantage sur l'analyse des trajectoires du système sous l'influence d'une perturbation de l'environnement : elle vise notamment à apprécier l'amplitude et la durée de la déformation et compare l'état initial du système à son état final après perturbation (figure 1).

Dans cette synthèse, nous nous appuierons sur ces deux approches afin de montrer en quoi et comment les systèmes d'élevage extensifs peuvent solliciter les capacités adaptatives des animaux et induire des réponses variées tant du point de vue de leur forme (robustesse, élasticité, flexibilité, plasticité), de leur support d'expression (physiologie/comportement) que de leur efficacité à court, moyen et long terme. Les exemples mobilisés pour parvenir à cette démonstration concernent les productions d'herbivores qui sont les plus représentées dans les systèmes extensifs.
La démarche retenue est progressive. Elle consiste dans un premier temps à caractériser les contraintes jugées spécifiques des systèmes extensifs et à identifier les qualités adaptatives que l'on peut attendre des animaux pour répondre à ces contraintes. Nous poursuivons par une analyse des réponses physiologiques et comportementales qui permettent aux animaux de s'adapter lorsqu'ils sont soumis à une gamme de niveaux de contraintes. Enfin, en nous appuyant sur des comparaisons de réponses entre races soumises à une même contrainte, nous proposons une interprétation des profils de réponse du point de vue des avantages adaptatifs qu'ils procurent.

\section{1 / Caractérisation des sys- tèmes d'élevage extensifs : identification des contrain- tes et des qualités adapta- tives attendues pour les ani- maux}

Selon Landais et Balent (1995), il n'est pas possible de définir dans l'absolu les systèmes d'élevage extensifs, mais leurs évolutions peuvent 
Figure 1. Représentation schématique de trajectoires adaptatives caractérisant des propriétés de robustesse, d'élasticité, de flexibilité ou de plasticité : variation au cours du temps de la réponse comportementale et/ou physiologique du système sous l'effet d'une contrainte (d'après Alcaras et Lacroux 1999).

\section{Systèmes indéformables}

\section{Robustesse}

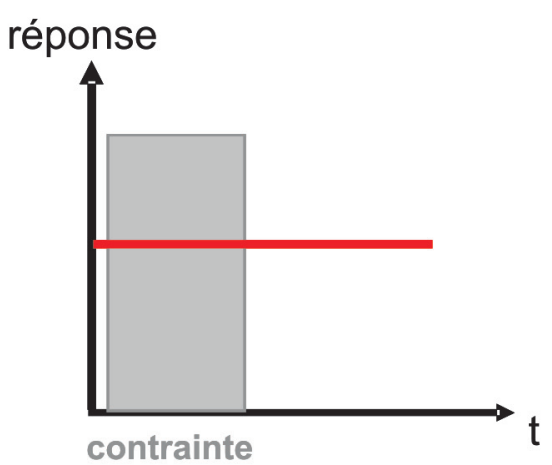

En l'absence de modification de trajectoire, les systèmes sont qualifiés de robustes ou de résistants. C'est leur résistance aux pressions extérieures qui leur permet de rester viables. Ces systèmes peuvent être détruits si la pression de l'environnement dépasse leurs capacités de résistance.

\section{Systèmes déformables}

\section{Elasticité / Flexibilité}

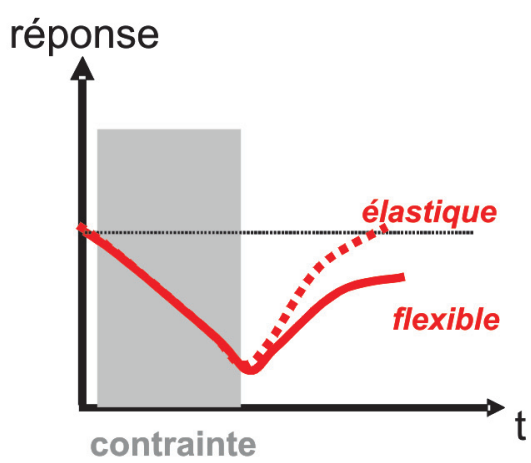

La flexibilité rend compte de la capacité des systèmes à se déformer sous l'effet d'une contrainte. Lorsque la déformation est réversible, elle est qualifiée d'élastique. Le système revient alors à son état initial lorsque la contrainte cesse d'agir. Lorsque la contrainte ou les changements de l'environnement induisent une déformation irréversible de la structure du système ou de ses finalités, l'adaptation est qualifiée de flexible. néanmoins être qualifiées sans ambiguïté : le concept d'extensification correspond à une réduction des facteurs de production mobilisés, à l'exception de la surface. La notion d'extensification, qu'elle s'applique à l'ensemble du système d'élevage ou seulement à une de ses parties, doit toujours être définie par rapport au(x) facteur(s) de production considéré(s). Classiquement, on se réfère au facteur terre qui fut longtemps le facteur de production limitant, mais d'autres facteurs (travail, capital d'exploitation, consommations intermédiaires) peuvent évidemment être pris comme référence (Landais et Balent 1995). Même s'il est difficile de qualifier ces systèmes, il est possible d'en préciser quelques caractéristiques.

Les systèmes d'élevage extensifs mobilisent de vastes espaces et se caractérisent par des niveaux de productivité animale modérés. La référence en ce domaine est constituée par les systèmes pastoraux et, dans une moindre mesure, par les systèmes herbagers. Les élevages pastoraux utilisent des parcours, surfaces peu productives que doivent parcourir les animaux pour ingérer une ration suffisante pour couvrir leurs besoins. En France, ces systèmes sont plutôt présents dans le Sud. Les systèmes herbagers reposent davantage sur la culture de l'herbe et se rencontrent plutôt dans le centre et le Nord de l'hexagone et dans les zones de moyenne montagne (Landais et Balent 1995). Dans les deux cas, l'exploitation de la surface fourragère est en majeure partie faite par les animaux qui pâturent des ressources plus ou moins abondantes et variées. Ces surfaces de végétation permanente ne peuvent guère être modifiées par des pratiques culturales et la marge d'amélioration ou de gestion des parcours est faible. Si l'éleveur fait le choix de limiter ses coûts de production, la capacité de ses animaux à se déplacer et à pouvoir tirer profit de ressources végétales variées est alors fortement sollicitée. Lorsque les surfaces fourragères sont très éloignées du siège d'exploitation, le troupeau est contraint à réaliser des déplacements sur de grandes distances. C'est le cas des systèmes transhumants. Certaines exploitations ovines préalpines (figure 2) pratiquent même une double transhumance : une transhumance estivale en altitude et une transhumance hivernale inverse qui se termine sur des parcours boisés de bords de mer (Debayle 2004). Le lot principal de brebis, conduit en un agnelage par

\section{Systèmes transformables}

\section{Plasticité}

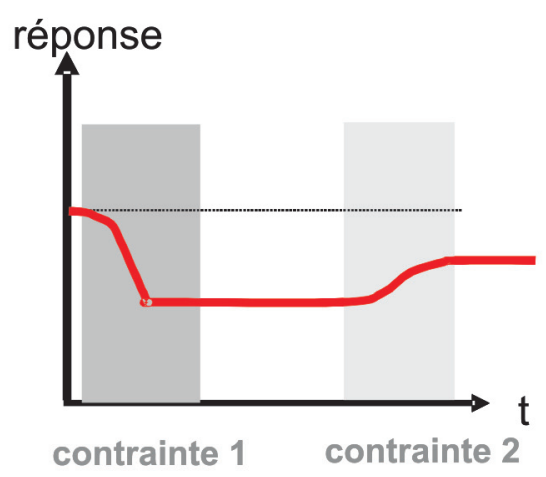

La plasticité est une propriété des systèmes vivants ayant achevé leur développement de remanier leur structure et enrichir leur répertoire réactionnel de sorte qu'ils développent de nouvelles facultés d'adaptation. Les systèmes plastiques sont des systèmes qui s'adaptent en se transformant et apprennent par là même à s'adapter. Un système capable de faire preuve de plasticité est capable à fortiori de faire preuve d'élasticité et de flexibilité.

an, est lutté sur les parcours de plaine. La gestation est assurée sur les alpages d'altitude puis les brebis reviennent pour agneler puis allaiter sur le siège de l'exploitation dans les Préalpes. Cet élevage utilise donc de vastes surfaces, pour partie en association avec d'autres éleveurs (1500 ha d'alpage, 2000 ha de parcours), et 120 ha de pâturage au niveau du siège de l'exploitation qui sont les seules parcelles mécanisables. $\mathrm{Au}$ cours de ses déplacements de transhumance, l'éleveur conduit son troupeau sur trois types de végétations dans trois milieux contrastés et parvient ainsi à alimenter ses brebis tout au long de l'année avec le pâturage. La productivité individuelle est faible mais elle est compensée par la grande taille du troupeau (600 à 800 brebis). Les charges sont modestes (déplacement et droit d'accès aux ressources pâturées) et le recours aux intrants est très limité.

Cette structuration de l'espace fourrager implique une cohérence entre les modalités d'utilisation de l'espace et les choix de conduite des animaux. Cette cohérence intègre les aptitudes particulières demandées aux animaux. Les systèmes extensifs se rencontrent dans les régions où la pression démographique 
Figure 2. Représentation schématique de la succession des milieux (zone géographique, type de végétation, altitude) utilisés par un troupeau de brebis Préalpes transhumant au cours d'un cycle de production (lutte/gestation/allaitement).

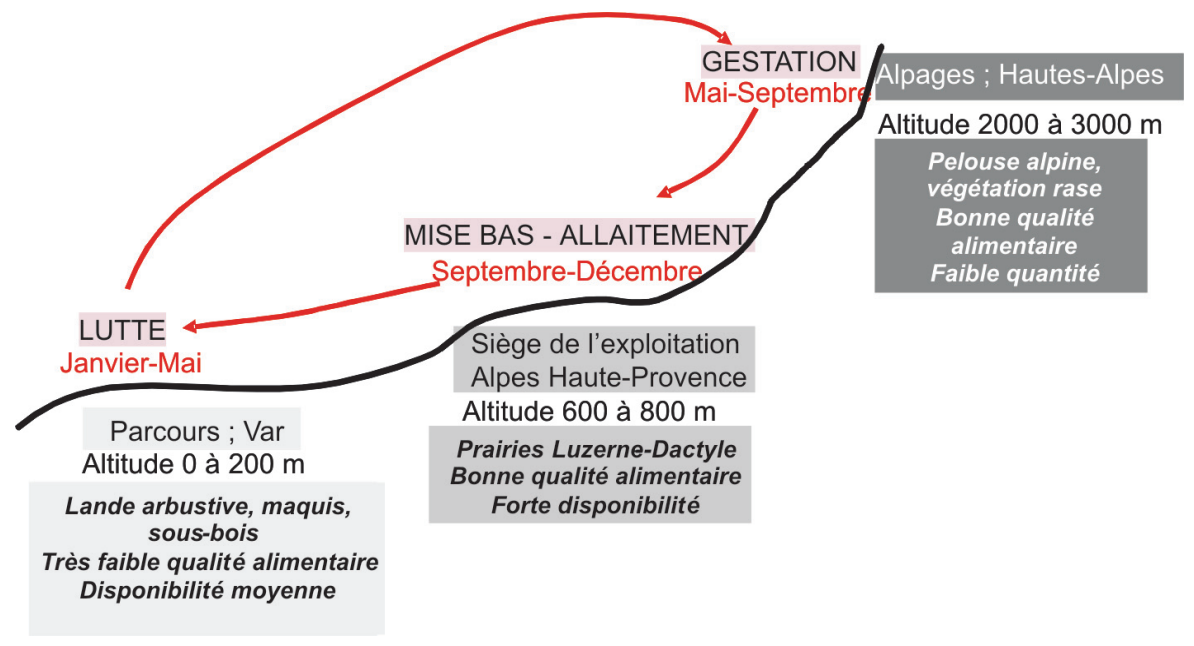

est faible, et donc où la terre est relativement disponible et pas trop chère. Les terres d'élevage extensif sont fréquemment des surfaces non ou peu mécanisables parce que pentues en montagne, peu portantes en zone humide, et pouvant comporter de nombreux obstacles. Ces espaces sont par ailleurs souvent soumis à des contrastes saisonniers et à des aléas climatiques qui affectent leur production de biomasse. Les animaux élevés dans les systèmes extensifs doivent de ce fait, être à la fois capables de valoriser les bonnes ressources fourragères, mais aussi de supporter avec un moindre coût zootechnique des périodes plus contraignantes. Cette aptitude passe par une capacité à constituer des réserves corporelles en période d'abondance et à pouvoir les mobiliser efficacement en période de disette. Une grande partie de la fonction de stockage-déstockage de ces élevages extensifs est dévolue aux animaux qui ne disposent pour ce faire que des variations de leur état corporel. Plus les systèmes d'élevage seront extensifs et dépourvus de capacité à faire des stocks fourragers pour suppléer la (les) mauvaise(s) saison(s), plus les animaux devront être efficaces dans leur aptitude à accroître leurs réserves corporelles et à les mobiliser («vache ou brebis accordéon»).

Pour parvenir à assurer leur ration au pâturage, les animaux doivent être capables de prospecter le milieu et d'y consommer des couverts parfois peu appétants. Cela signifie qu'ils aient de bonnes aptitudes à la marche et à l'exploration y compris dans des espaces à fortes contraintes physiques (pente, barres rocheuses...), mais aussi qu'ils soient capables d'apprentissage (inges- tion de nouveaux fourrages) et de mémorisation de la localisation des espaces les plus riches (Dumont et al 2001). Pilotées judicieusement par l'éleveur (Meuret 1993), de telles capacités permettent de valoriser au mieux le territoire disponible plutôt que de s'en tenir à quelques circuits rapidement surpâturés, et contribuent ainsi à préserver le territoire et ses ressources sur le long terme (Debayle 2004).

En conditions d'élevage extensif particulièrement sévères, les contraintes se cumulent dans la mesure où il n'y a aucun stock récolté et redistribué et où les élevages ne disposent souvent d'aucun encadrement technico-commercial ou de système d'assurance. Brunschwig (1992) a ainsi observé que les systèmes d'élevage extensifs des Andes centrales, localisés dans la puna à plus de $4000 \mathrm{~m}$ d'altitude et qui ne peuvent compter que sur la solidarité locale, visaient avant tout à se maintenir d'une année sur l'autre plutôt qu'à accroître leur production. Dans de telles conditions, l'aptitude des femelles adultes à maintenir leur reproduction est fondamentale car elle garantit la capacité du système à reconstituer les effectifs des troupeaux au lendemain d'épisodes de forte mortalité ou de réformes importantes. Cette capacité des femelles à se reproduire est donc essentielle dans ces systèmes d'élevage très extensifs. Elle correspond de fait à deux aptitudes spécifiques : celle de préserver une fécondité des adultes, et celle d'allaiter et de protéger le ou les jeune(s) en condition difficile, notamment de sous-alimentation. Ces capacités permettent aux élevages les plus extensifs de maintenir une reproduction et une production minimales de lait ou de viande même les années difficiles.
En France métropolitaine, les contraintes d'élevage sont bien sûr moindres, mais l'implication fréquente des élevages extensifs ou en voie d'extensification dans des filières de qualité les conduit à respecter des cahiers des charges limitant fortement les solutions connues pour atténuer les effets de certaines contraintes. Ainsi, l'obligation d'atteindre une relative autonomie alimentaire (Appellation d'Origine Protégée AOP Saint-Nectaire) ou de limiter les quantités de concentré distribuées (AOP Laguiole, Comté...) peut affecter le niveau alimentaire des animaux ou leur niveau de production (Blanc et al 2004). De fait, ces élevages sont eux aussi amenés à supporter les aléas et à assurer par eux-mêmes les conditions de leur pérennité.

En résumé, les systèmes extensifs sont très dépendants de l'environnement et de ses variations du fait de leur faible niveau d'artificialisation (maintien des coûts de production à un faible niveau, recours limité aux intrants, absence de bâtiment). La maîtrise de l'environnement par l'éleveur est extrêmement limitée de sorte que ces systèmes reportent une part de leur aptitude à subir des contraintes sur les animaux. Dans de tels systèmes, la collecte de la ressource alimentaire est en majeure partie confiée à l'animal. Du fait de la variabilité et de l'hétérogénéité des ressources alimentaires, les qualités adaptatives attendues de la part des herbivores sont une capacité à maintenir un niveau de production dans des conditions de dynamiques nutritionnelles variables, une aptitude à explorer l'espace et à évoluer dans l'utilisation des ressources alimentaires, et une faculté à assurer la pérennité du troupeau c'est-à-dire à enchaîner régulièrement des cycles de reproduction.

\section{2 / Caractérisation des réponses de production et de reproduction des rumi- nants soumis à des dyna- miques nutritionnelles va- riables}

\section{1 / A l'échelle de la perfor- mance}

Les réponses des ruminants à une restriction alimentaire ont largement été étudiées et ont fait l'objet de plusieurs synthèses bibliographiques (Chilliard et al 1998a et b, Blanc et al 2006). Les études portant sur les capacités adaptatives des ruminants à des restrictions alimentaires révèlent le rôle central joué par les réserves adipeuses. Leur capacité à se mobiliser et à se reconstituer 
confèrent des propriétés d'élasticité à l'organisme lorsque celui-ci est soumis à des phases de restriction alimentaire puis de réalimentation. Ainsi, Atti et Bocquier (1999) ont observé que des brebis Barbarine sous-alimentées à $20 \%$ de leurs besoins pendant 22 semaines puis réalimentées à $150 \%$ des besoins d'entretien pendant 23 semaines parvenaient au même poids et à une composition corporelle identique à celle des brebis témoin maintenues à poids constant pendant les 45 semaines d'essai. Une telle réponse peut être qualifiée d'élastique (figure 1) dans la mesure où l'organisme retrouve un état final après réalimentation semblable à celui qui le caractérisait avant la perturbation. Une telle élasticité permet d'assurer une économie sur les apports énergétiques totaux puisque dans le cas de la séquence alimentaire variable, ceux-ci (156 UFL/brebis) ont été inférieurs de $20 \%$ à ceux de la séquence nutritionnelle stable (188 UFL/brebis). L'économie réalisée correspond à 1,7 mois de couverture des besoins d'entretien. Elle peut s'interpréter par un accroissement de l'efficacité alimentaire résultant directement des mécanismes de mobilisation et d'épargne mis en œuvre pendant la sous-alimentation. Les adaptations digestives, hormonales et métaboliques qui soutiennent de telles réponses ont été décrites par Chilliard et al (1998a, 2000).

Des profils de réponses de type «rebond» révélant une capacité du système à se déformer temporairement sous l'effet de la contrainte alimentaire (propriété d'élasticité et de flexibilité, figure 1) s'observent également chez les jeunes animaux en croissance. Le processus est alors qualifié de croissance compensatrice. Il est mis en évidence lors d'un retour à une alimentation non limitante (pâturage) suite à une période de restriction alimentaire (le plus souvent hivernale). Cette réponse a été révélée dans des situations où les animaux sont alimentés à volonté avec des fourrages de différentes qualités (Hoch et al 2005), ou encore dans des études où les animaux subissent une phase de restriction alimentaire (quantités offertes limitées, Sainz et al 1995, Blanc et al 2007 ; ou ration de faible densité énergétique, Tolkamp et al 2006) suivie d'une réalimentation. Les travaux menés sur la croissance compensatrice indiquent, chez les ovins (figure 3a) comme chez les bovins, qu'à même niveau de réalimentation, la réponse est d'autant plus forte que la durée et la sévérité de la restriction préalable ont été importantes. Elle est par ailleurs modulée par l'âge des animaux.

Les principaux mécanismes connus impliqués dans la croissance compensatrice sont décrits dans la revue de Hoch et al (2003). Le premier phénomène observé est une augmentation significative des quantités ingérées lors d'une phase de retour à une alimentation non limitante qui succède à une période de restriction alimentaire. Hoch et al (2005) ont ainsi observé un accroissement des quantités ingérées durant la période estivale (alimentation à volonté avec du foin de très bonne qualité) deux fois plus important chez des génisses Salers nourries en hiver avec du foin de mauvaise qualité que chez leurs congénères ayant toujours été alimentées avec $\mathrm{du}$ foin de bonne qualité. Rapporté au poids vif cela correspond à un écart d'ingestion de $10 \%$ entre les lots. Ferrer Cazcarra et Petit (1995) ont observé que lorsque la croissance compensatrice s'opère au pâturage, des génisses Charolaises contraintes pendant l'hiver sont capables de rattraper une partie de leur retard de croissance en ingérant davantage. Cette réponse est principalement permise par une augmentation du temps de pâturage journalier en situation de pâturage non limitant. Tolkamp et al (2006) ont par ailleurs montré, chez les ovins, que les niveaux d'ingestion supérieurs observés chez les animaux ayant subi une restriction alimentaire peuvent s'expliquer en partie par leur faible état corporel.

Le second mécanisme s'appuie sur une diminution du métabolisme de base et de l'activité physique pendant la phase de restriction alimentaire. Celleci se traduit par une baisse des dépenses énergétiques due en grande partie à une réduction de la taille et du poids des tissus (splanchniques notamment) et à une modification des dépenses énergétiques de tout ou partie des tissus (baisse du débit sanguin). Le métabolisme énergétique et protéique s'adapte en parallèle à cette baisse des flux de nutriments pour préserver le compartiment protéique

Figure 3. Évolution des poids vifs (a) et de la leptinémie (b) d'agnelles Mérinos d'Arles soumises à des niveaux alimentaires différenciés au cours des 6 mois post-sevrage puis réalimentées à volonté (d'après Blanc et al 2007).

(a) Poids vifs ( $\mathrm{kg}$ )

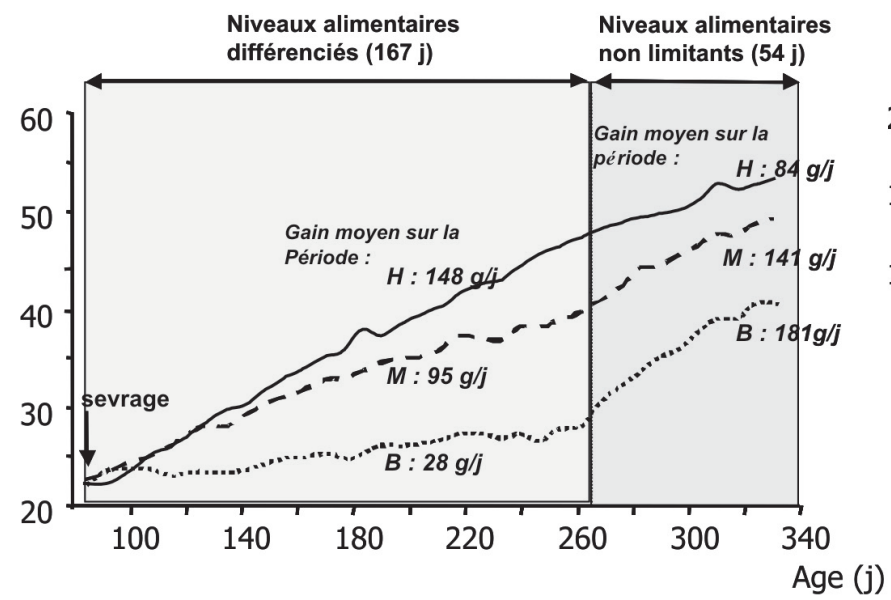

(b) Leptinémie plasmatique $(\mathrm{ng} / \mathrm{mL})$

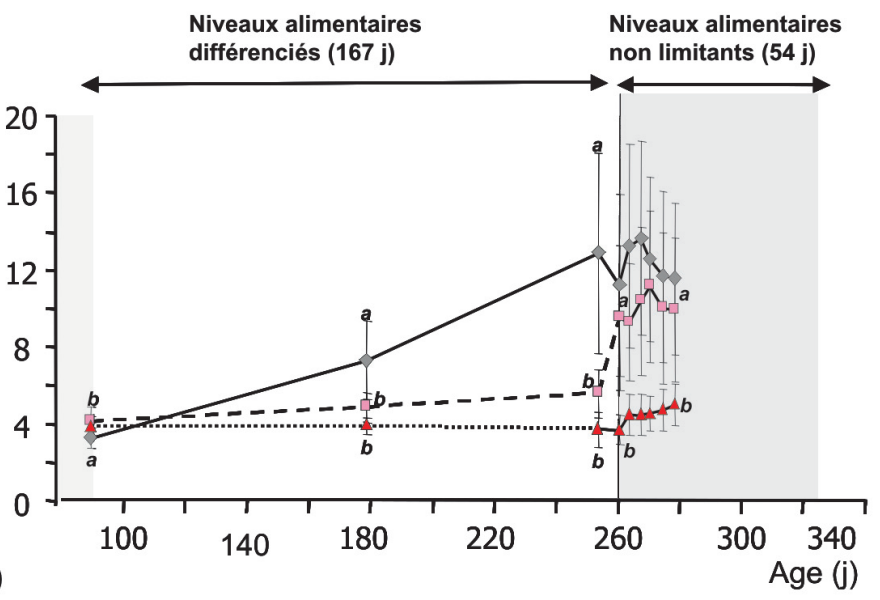

Lot $\mathbf{H}(n=10)$ : agnelles nourries à volonté au cours des 9 mois suivant le sevrage

Lot $\mathbf{M}(n=10): 80 \%$ des besoins énergétiques couverts au cours des 6 mois pōst-sevrage puis alimentation à volonté les 3 mois suivants.

...... Lot $\mathrm{B}(n=10): 62 \%$ des besoins énergétiques couverts au cours des 6 mois post-sevrage puis alimentation à volonté Les 3 mois suivants. 
aux dépens des réserves adipeuses. Lors de la réalimentation, le métabolisme basal de l'animal reste bas, avant de réaugmenter lentement. Durant cette période de transition, l'efficacité d'utilisation de l'énergie et la proportion de protéines du régime retenue sont accrues alors que les besoins énergétiques pour la croissance sont maintenus à un niveau bas (Hoch et al 2003).

Ces deux mécanismes mettent en jeu des modifications hormonales. Chez les ruminants, la sous-alimentation s'accompagne d'adaptations métaboliques et hormonales qui ont été décrites par Chilliard et al (1998a) : baisse des concentrations circulantes en insuline, leptine, hormones thyroïdiennes, IGF-1 et augmentation des taux circulants de glucocorticoïdes et de GH. Pendant la réalimentation ces évolutions sont rapidement inversées et contribuent à une meilleure utilisation des nutriments pour l'anabolisme et la croissance. Les adaptations digestives et métaboliques en jeu dans la phase de réalimentation sont effectives en quelques jours (Hoch et al 2003), ce qui témoigne de la réactivité de l'organisme à identifier et mettre à profit une amélioration de son état nutritionnel.

Les mécanismes adaptatifs précédemment cités permettent de soutenir des profils de réponses que l'on peut qualifier d'élastiques lorsque le poids et/ou la composition corporelle finale des animaux ayant subi les restrictions alimentaires sont très proches de ceux des animaux n'ayant pas subi de contrainte nutritionnelle. Cette élasticité est observée par Hoch et al (2005) sur des génisses Salers ayant subi une sous-alimentation (foin de mauvaise qualité) durant deux hivers successifs et dont la composition corporelle finale est identique à celle des génisses alimentées en continu avec du foin de bonne qualité (lot $\mathrm{C}$, figure 4). Au final, ce profil de réponse élastique s'explique pour partie par une augmentation temporaire du niveau d'ingestion et pour partie par l'amélioration de l'efficacité alimentaire. Ainsi, à même poids et composition corporelle finaux, les génisses ayant eu une croissance discontinue (lot D) ont consommé 4510 UFL contre 4905 UFL pour celles en croissance continue (lot $\mathrm{C}$ ), ce qui correspond à un accroissement de l'efficacité d'utilisation de l'énergie de $8 \%$. Les réponses adaptatives associées à des cycles de sous-alimentation/réalimentation mettent ainsi en évidence l'aptitude de l'organisme à mettre à profit une situation nutritionnelle favorable avec plus d'efficacité lorsqu'elle succède à une restriction alimentaire.

Lorsque la compensation n'est que partielle à l'issue de la phase de réali-
Figure 4. Évolution des poids vif vides, des poids de carcasse, des poids de muscles et de tissu adipeux ( \pm erreur standard) de génisses Salers nourries à volonté avec du foin de mauvaise qualité pendant l'hiver et du foin de bonne qualité pendant l'été (lot $D, \mathrm{n}=14$ ) vs nourries en continu avec du foin de bonne qualité (lot $C$, $\mathrm{n}=9$ ). (d'après Hoch et al 2005).

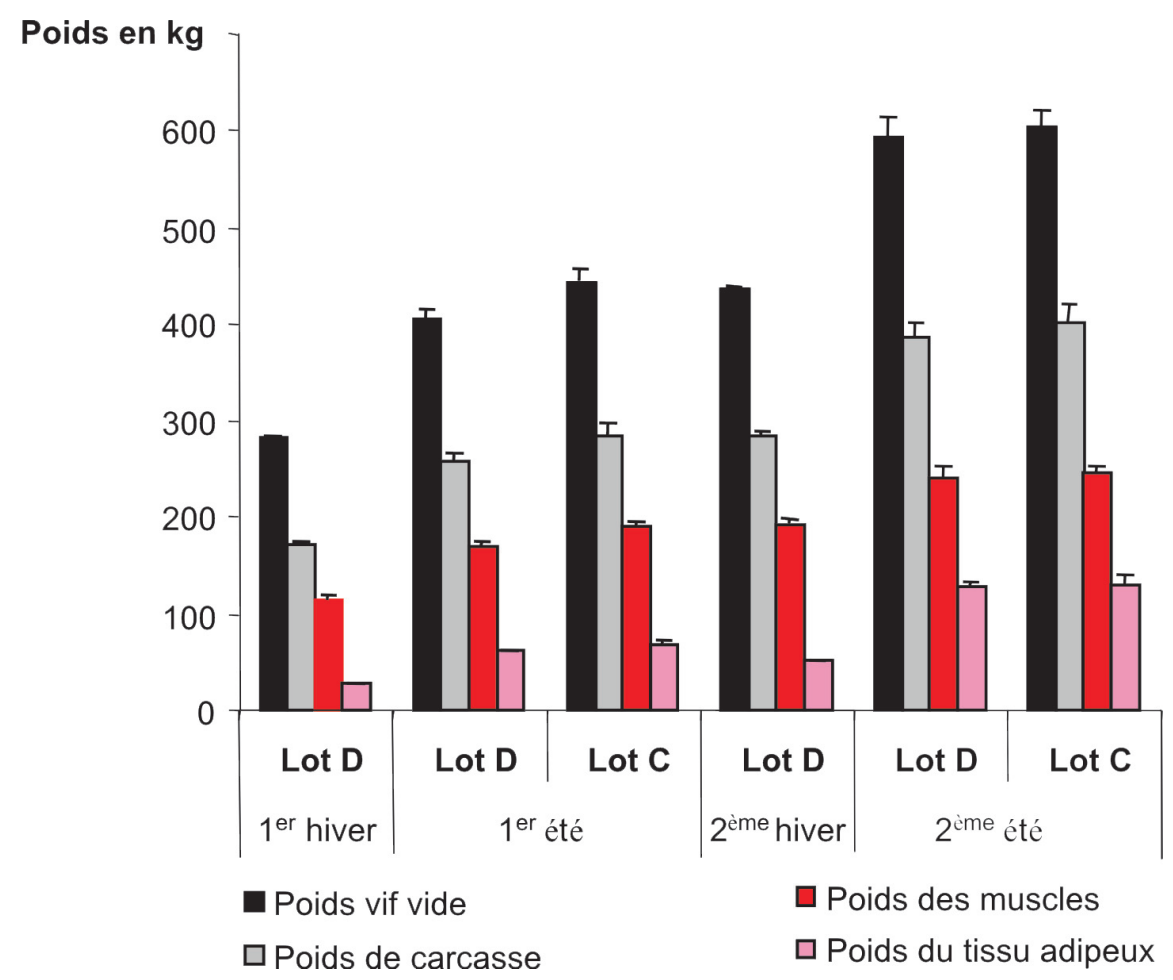

mentation (poids et composition corporelle différents de ceux observés avant l'application de la contrainte alimentaire), la réponse est qualifiée de flexible. Certains travaux montrent en effet que la composition corporelle des animaux sous-alimentés peut être durablement modifiée même après une phase de réalimentation. C'est particulièrement le cas lorsque la restriction est très intense et intervient dans le très jeune âge. Elle peut alors modifier le développement squelettique de l'animal. Un tel profil de réponse a été observé sur les agnelles Mérinos d'Arles fortement sous-alimentées (lot B) qui ont présenté, après 3 mois de réalimentation, des états corporels (lot $\mathrm{H}: 3,6 \pm 0,2$; lot $\mathrm{M}$ : $3,6 \pm 0,2 ; \operatorname{lot} \mathrm{B}: 2,6 \pm 0,2)$ et des leptinémies (indicateur d'adiposité, figure 3b) significativement plus faibles que ceux des agnelles témoins (lot $\mathrm{H}$ ) ou faiblement sous-alimentées (lot M).

Dans la mesure où le phénomène de croissance compensatrice s'observe généralement sur des animaux qui n'ont pas achevé leur développement, il est préférable de parler de flexibilité (au sens large) plutôt que de plasticité (figure 1) pour qualifier le processus d'adaptation en jeu. La plupart des travaux ne se poursuivent pas sur un temps suffisamment long pour pouvoir déterminer si la réponse adaptative d'animaux soumis à des restrictions alimentaires dans leur jeune âge les rend plus aptes à s'adapter à des sous-alimentations ultérieures. Il est cependant possible d'imaginer que l'amplitude de la sous-alimentation et sa durée puissent induire des adaptations structurelles et physiologiques de l'organisme telles que celui-ci devienne plus efficace du point de vue de son aptitude à faire face à de nouvelles sous-alimentations (plasticité).

\section{2 / Du point de vue de la prio- rité donnée à l'investissement reproductif}

Dans les systèmes d'élevage extensifs la fonction de reproduction soutient non seulement la pérennité des élevages, mais permet aussi d'assurer une part de leur productivité (jeunes, production laitière). De nombreuses études ont révélé la sensibilité de cette fonction biologique à l'état nutritionnel de la femelle (Robinson et al 2006), mais peu ont cherché à intégrer les connaissances acquises dans un cadre de réflexion permettant de raisonner l'effet combiné des réserves corporelles et de la dynamique de l'état nutritionnel de la femelle sur sa capacité à s'investir plus ou moins rapidement dans un nouveau cycle de production (Friggens 2003, Friggens et al 2010).

Les effets de l'alimentation sur l'aptitude des femelles à se reproduire sont 
observés aux différentes phases de la vie productive. Le développement (proportion du poids adulte) et le statut nutritionnel conditionnent ainsi le moment d'apparition de la puberté chez les jeunes femelles (Foster et al 1985, Suttie et al 1991, Le Cozler et al 2008). Il influence également plusieurs composantes de la performance de reproduction chez l'adulte : cyclicité, fertilité, prolificité, comportement sexuel (Butler 2003, Diskin et al 2003). Les effets de la sous-alimentation sur la reproduction se traduisent le plus fréquemment par l'apparition de délais (retard dans la reprise de cyclicité post-partum), par des mises en veille temporaires (interruption de cyclicité) ou par une baisse des performances (moindre prolificité).

Les délais interviennent essentiellement dans les phases de démarrage (puberté) ou de redémarrage (reprise de cyclicité post-partum ou saisonnier) de l'activité sexuelle. Ainsi, une sous-alimentation subie pendant la phase de croissance post-sevrage s'accompagne d'un retard d'apparition de la puberté chez des génisses Charolaises (Dozias et al 2006) comme chez des agnelles Merinos d'Arles (Blanc et al 2007). La sous-alimentation ne semble avoir un effet sur l'acquisition de la puberté qu'en situation de forte restriction. Ainsi, chez les agnelles non pubères, des retards d'apparition de la puberté ont été constatés uniquement dans le lot le plus sévèrement sous-alimenté

Figure 5. Évolution de la proportion (\%) d'agnelles pubères au sein de lots soumis à des niveaux alimentaires différenciés depuis le sevrage (3 mois) jusqu'à 9 mois puis réalimentées à volonté.

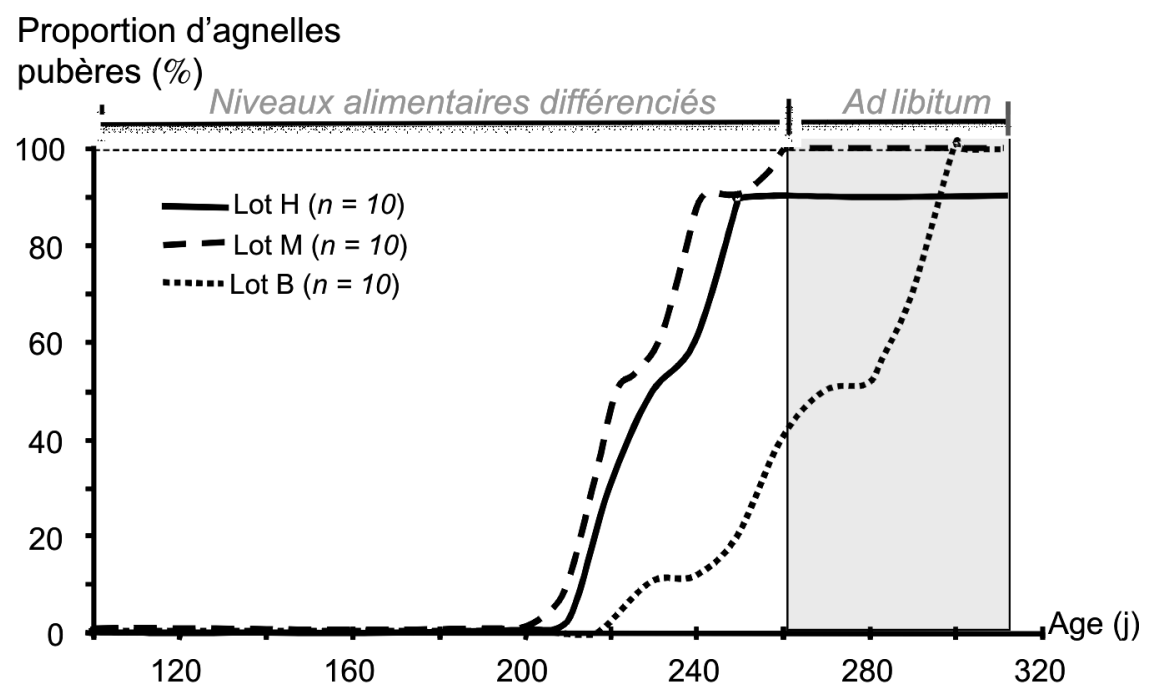

Lot $\mathbf{H}(n=10)$ : agnelles nourries à volonté au cours des 9 mois suivant le sevrage

Lot $\mathbf{B}(n=10): 62 \%$ des besoins énergétiques couverts au cours des 6 mois post-sevrage puis alimentation à volonté les 3 mois suivants.

Lot $\mathbf{M}(n=10): 80 \%$ des besoins énergétiques couverts au cours des 6 mois post-sevrage puis alimentation à volonté les 3 mois suivants.

(lot B : $62 \%$ des besoins énergétiques couverts, figure 5) tandis que l'âge à la puberté $(225 \pm 14 \mathrm{j})$ des agnelles faiblement sous-alimentées (lot $\mathrm{M}: 80 \%$ des besoins énergétiques couverts) n'a pas différé de celui du lot témoin alimenté à (lot $\mathrm{H}: 230 \pm 14 \mathrm{j}$ ). Ce résultat révèle la capacité de l'animal à acquérir une aptitude à se reproduire même en situation nutritionnelle non optimale. Certaines agnelles $(n=4)$ du lot B pendant la parvenues à devenir pubères taire à un âge moyen de $247 \pm 14 \mathrm{j}$ et un poids moyen de $28,6 \pm 2,3 \mathrm{~kg}$. Ces individus étaient les plus lourds du lot $(+1 \mathrm{~kg}$ en moyenne par rapport aux autres) et avaient les états corporels les plus élevés (note d'état corporel : $+0,3$ sur une échelle de 1 à 5). Les autres puberté dans les 3 semaines suivant début de la réalimentation à un âge moyen de $287 \pm 11 \mathrm{j}$ et un poids moyen (1,1 kg. Il semblerait donc qu'à partir du moment où la femelle a atteint une certaine maturité (âge, poids, et adiposité minimum), l'amélioration des conditions nutritionnelles permette le déclenchement de la fonction de reproduction dans des délais relative-

De tels délais dans l'activation du fonctionnement ovarien sont également observés chez des femelles pubères lors de la reprise de cyclicité post-partum. Plusieurs travaux rapportent un allonge- 
s'opère au moment de l'émergence d'une nouvelle vague folliculaire et chez des femelles émaciées.

Ces différents processus adaptatifs permettent à l'organisme d'opérer, à un moment donné du cycle de production, des arbitrages entre des priorités qui assurent sa propre survie et d'autres qui sont orientées vers la survie du ou des jeunes (investissement maternel), et/ou l'investissement dans la génération future (Friggens 2003). La régulation de telles priorités est particulièrement importante chez les espèces à cycle long pour lesquelles la conception d'une nouvelle génération (fonction de reproduction) s'opère à un moment où l'orientation des nutriments privilégie la survie de la génération actuelle (fonction de lactation). En effet, l'initialisation d'une gestation peut être risquée pour la survie de la femelle si les apports nutritionnels et/ou si ses réserves corporelles sont insuffisantes. Au cours du dernier tiers de la gestation les besoins énergétiques de la femelle s'accroissent de façon importante par rapport aux besoins d'entretien $(+30$ à $40 \%$ chez la vache et +50 à $80 \%$ chez la brebis et la chèvre) alors que sa capacité d'ingestion reste relativement stable (INRA 2007). La mise bas génère ensuite une nouvelle dépense énergétique par l'enclenchement de la lactation. Selon le potentiel laitier de la femelle (type allaitant vs laitier) les besoins énergétiques liés à la production laitière peuvent représenter de 0,7 (vache allaitante produisant $8 \mathrm{~kg}$ de lait/j) à plus de 4 fois (vache laitière produisant $40 \mathrm{~kg}$ lait/j) les besoins énergétiques d'entretien. Ainsi, la réussite de la reproduction implique pour la femelle une forte capacité à supporter ultérieurement l'accroissement de ses besoins nutritionnels. Les régulations de la reproduction par l'état nutritionnel suggèrent qu'à un moment donné, l'organisme est capable d'intégrer l'état du bilan nutritionnel et des réserves corporelles. Une telle évaluation à des phases clé du processus reproductif pourrait constituer un moyen de remettre en cause l'engagement de la femelle dans une nouvelle gestation, et de limiter ainsi le risque associé à la reproduction. Si les signaux métaboliques et hormonaux susceptibles de renseigner l'organisme sur son statut nutritionnel sont relativement bien identifiés, les mécanismes par lesquels ces signaux modulent la réponse reproductive sont quant à eux encore mal connus.

\section{3 / A l'échelle de la carrière des femelles}

L'efficacité des processus d'adaptation peut s'apprécier sur le long terme en considérant la longévité des femelles reproductrices dans des conditions d'élevage où elles subissent des situations d'alimentation contraignantes à certaines périodes, et notamment dans le jeune âge. Une telle démarche a été menée sur 217 génisses Charolaises élevées de 3,5 mois à 3 ans (âge au 1 er vêlage) selon trois niveaux d'alimentation (H, M, B) appliqués en bâtiment pendant l'hiver et au pâturage (chargements variables) durant l'été (Dozias et al 2006). Au premier vêlage, les vaches différaient au maximum de $100 \mathrm{~kg}$ de poids vif et de 1,5 point de note d'état corporel (échelle de 1 à 5). Après le vêlage, elles ont été conduites durant quatre lactations successives selon deux modalités alimentaires ( $\mathrm{h}:$ couverture des besoins énergétiques $v s \mathrm{~b}$ : besoins énergétiques - 2 UFL) puis ont été abattues. Au total, quatre modalités générant des trajectoires alimentaires différentes ont ainsi été analysées ( $\mathrm{Hh}, \mathrm{Hb}, \mathrm{Mb}, \mathrm{Bb}$, environ 50 individus/lot). La survie des vaches a été estimée par le nombre d'animaux ayant été capables de mener à bien les quatre lactations et par l'analyse des causes de réformes, sachant qu'une vache vide ou ne pouvant pas vêler dans la fourchette de dates prévues était systématiquement réformée.

La compensation des écarts de poids initialement créés entre lots est, en quatrième lactation, soit totale (lots $\mathrm{Bb} v s$ $\mathrm{Mb}$ ), soit nulle (lots $\mathrm{Bb} v s \mathrm{Hh}$ ) (figure 6). Une femelle introduite dans le dispositif a sevré en moyenne 2,5 veaux (sur 4 lactations potentielles). La productivité du lot Hh (2,7 veaux sevrés) n'a été que très légèrement supérieure à cette valeur (tableau 1). A l'issue de la quatrième lactation, et compte tenu de la politique de réforme, c'est dans le lot $\mathrm{Bb}$ que l'on observe le taux de survie le plus important à 7 ans (49 contre $42 \%$ en moyenne pour les autres lots). Cette réponse se vérifie pour chacune des sept cohortes analysées. Les réformes précoces ont surtout été le fait de vaches n'ayant pas vêlé $(46 \%$ des causes de réforme) ou ayant perdu précocement leurs veaux (4\%). C'est dans le traitement $\mathrm{Bb}$ que ces deux problèmes ont été les moins fréquents $(35 \%$ pour les réformes associées à une non gestation et $2 \%$ pour les réformes liées à la mortalité des veaux). En revanche, les vaches de ce lot semblent plus fragiles que celles des autres lots puisque le taux de perte pour causes accidentelle ou sanitaire (mort, fractures, maladies) y est deux fois plus élevé (14 vs 7\% ; tableau 1). Les vaches du lot $\mathrm{Bb}$ semblent traverser plus difficilement l'épreuve de la première lactation, mais une fois celle-ci passée elles apparaissent mieux adaptées aux conditions d'élevage subies (taux de réforme moins important que dans les autres lots). Cet exemple illustre une réponse qui peut être qualifiée de plastique dans la mesure où les individus du lot $\mathrm{Bb}$ qui survivent semblent avoir acquis des facultés adaptatives leur assurant une viabilité supérieure par rapport aux vaches ayant connu des conditions d'alimentation plus favorables durant leur phase de croissance.

Les exemples choisis ont permis de décrire des mécanismes d'adaptation physiologique mis en œuvre par les animaux subissant des épisodes de sousalimentation. A ces processus physiologiques et métaboliques s'ajoutent des adaptations comportementales qui contribuent à l'aptitude des herbivores à s'alimenter dans des conditions contraignantes de pâturage. Nous les détaillons

Figure 6. Poids moyens en 1ère et 4ème lactation de vaches Charolaises ayant subi des niveaux alimentaires différents $(H, M, B)$ durant la phase d'élevage (3,5 mois 1 er vêlage) puis deux niveaux alimentaires différenciés $(h, b)$ durant la phase de production (1ère à 4ème lactation) (d'apres Dozias et al 2006).
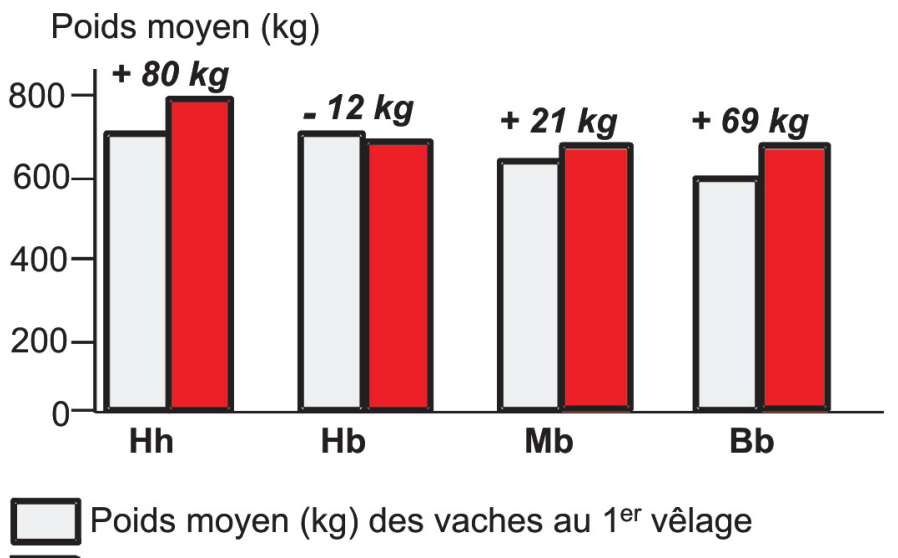

$\square$ Poids moyen $(\mathrm{kg})$ des vaches au $4^{\text {ème }}$ vêlage 
Tableau 1. Productivité, taux de survie et causes de réforme de vaches Charolaises ayant subi des niveaux alimentaires différents $(H, M, B)$ durant la phase d'élevage (3,5 mois - 1er vêlage) puis deux niveaux alimentaires différenciés ( $h, b)$ durant la phase de production (1ère à 4ème lactation) (D'après Dozias et al 2006).

\begin{tabular}{|l|c|c|c|c|}
\hline $\begin{array}{l}\text { Niveaux alimentaires appliqués durant la } \\
\text { phase d'élevage puis durant la phase de } \\
\text { production }\end{array}$ & $\mathbf{H h}$ & $\mathbf{H b}$ & $\mathbf{M b}$ & $\mathbf{B b}$ \\
\hline Nombre initial & 49 & 47 & 46 & 48 \\
Nombre de vêlages/génisse introduite & 2,7 & 2,5 & 2,2 & 2,7 \\
Age moyen des génisses à la réforme (mois) & 64 & 62 & 58 & 64 \\
Taux de survie à 80 mois (\%) & $45^{\mathrm{b}}$ & $42^{\mathrm{b}}$ & $39^{\mathrm{c}}$ & $49^{\mathrm{a}}$ \\
Causes de réformes & & & & \\
Vaches vides + avortements (\%) & $44^{\mathrm{b}}$ & $45^{\mathrm{b}}$ & $50^{\mathrm{b}}$ & $35^{\mathrm{a}}$ \\
Pertes (\%) & $7^{\mathrm{b}}$ & $7^{\mathrm{b}}$ & $7^{\mathrm{b}}$ & $14^{\mathrm{a}}$ \\
Tarissement précoce (\%) & 4 & 7 & 5 & 2 \\
\hline
\end{tabular}

Lettres différentes par ligne a, b différence significative au seuil de $5 \%$.

plus particulièrement dans la partie suivante.

\section{3 / Réponses comportemen- tales des herbivores face à une ressource fourragère hétérogène et variable}

$\mathrm{Au}$ pâturage, les herbivores domestiques ont la capacité de moduler leur comportement alimentaire pour s'adapter aux variations de disponibilités alimentaires des couverts qu'ils exploitent. Les quantités ingérées journalières sont classiquement décomposées comme étant le produit du temps de pâturage multiplié par le flux d'ingestion, luimême étant le produit de la fréquence de préhension par la masse moyenne des bouchées (Allden et Whittaker 1970). Lorsque la hauteur ou la densité des couverts pâturés diminue, la masse des bouchées diminue également (y compris sur prairies tropicales, Boval et al 2007), et les animaux augmentent leur fréquence de préhension et leur temps de pâturage afin de compenser les effets de cette diminution de disponibilité alimentaire sur les quantités ingérées. Ce phénomène a été largement décrit chez les ovins pâturant des monocultures de ray-grass en pâturage continu (Penning et al 1991) ou en pâturage tournant (Penning et al 1994). Les limites à cette compensation sont liées, en ce qui concerne la fréquence des bouchées au temps nécessaire à la préhension et à la mastication de l'herbe prélevée. Ces mouvements de mâchoires sont exclusifs chez les ovins alors qu'ils peuvent être mixtes chez les bovins (Prache et Peyraud 1997). Pour le temps de pâturage journalier, les possibilités de compensation sont limitées par le temps nécessaire à la satisfaction des besoins des animaux qui est plus élevé chez les animaux à forts besoins (Petit et Bechet 1995, Gibb et al 1999), par le temps nécessaire à la rumination et au repos, ou par une perte de motivation des animaux lorsque l'herbe pâturée devient très rase (Penning et al 1991).

Les mêmes ajustements du comportement d'ingestion peuvent être observés lorsque les animaux exploitent des couverts prairiaux hétérogènes. Ainsi, lorsque des groupes de cinq brebis exploitent deux parcelles de dactyle de différente taille, l'application de ces deux niveaux de chargement se traduit par une évolution différente de la structure du couvert et de sa valeur nutritive, avec en particulier une accumulation d'herbe épiée, puis sénescente au faible chargement. Au printemps, les brebis réalisent des niveaux d'ingestion élevés en compensant la diminution de disponibilité alimentaire au fort chargement par une augmentation de leur temps de pâturage journalier (Garcia et al 2003). En été et en automne, le prélèvement des animaux est contraint au fort chargement par une diminution du flux d'ingestion, en particulier de la masse des bouchées. Au faible chargement, il l'est avant tout par une diminution de la digestibilité globale du couvert. Les brebis réduisent alors leurs quantités ingérées journalières, les maintenant cependant au niveau de leur besoin d'entretien grâce au prélèvement sélectif des limbes verts au sein de l'horizon pâturé (Garcia et al 2003).

Sur parcours, des brebis sont également capables de maintenir leurs quantités ingérées journalières en sélectionnant une plus large gamme de bouchées en fin de séjour dans un parc (Agreil et al 2005). Les bovins aussi modulent leur comportement d'ingestion et leurs tactiques de recherche alimentaire en réponse aux variations de structure du couvert. Lorsque le chargement croît, des génisses augmentent leur temps de pâturage journalier, leur fréquence de préhension, le nombre de bouchées prélevées par pas (figure 7), et leur distance inter-individuelle (Dumont et al 2007). En fin de saison, la flexibilité comportementale des animaux se traduit au plus faible chargement par un maintien de la sélectivité alimentaire des animaux vis-à-vis des repousses végétatives de haute valeur nutritive, qui révèle une tactique d'exploration et de tri. Aux forts chargements, les distances parcourues quotidiennement diminuent en fin de saison, ce qui, couplé à une augmentation du nombre de bouchées prélevées par pas, révèlerait plutôt une stratégie de type «tondeuse».

Dans cette expérience les quantités ingérées journalières n'ont pas été mesurées mais les évolutions rigoureusement identiques du gain de poids vif quotidien (GMQ ; figure 7) et de la note d'état corporel des animaux entre les trois niveaux de chargement laissent présager que cette flexibilité du comportement alimentaire des animaux a été suffisante pour qu'ils maintiennent leur ingestion de nutriments (Dumont et al 2007). Plusieurs travaux réalisés sur des ressources végétales de différente nature rapportent d'ailleurs une grande stabilité de la qualité du régime sélectionné par les bovins alors que des gradients de chargement importants sont appliqués sur différents types de couverts (Ortega et al 1997, Schlegel et al 2000, Mayer et al 2003, Isselstein et al 2007 ; voir aussi Garcia et al 2003 pour les ovins et Fleurance et al 2009 pour les chevaux). Ainsi, la capacité des herbivores domestiques à moduler leurs tactiques comportementales contribue à la grande robustesse de leurs performances zootechniques au pâturage, et cela même lorsque les modes de gestion des prairies se traduisent par des disponibilités alimentaires très contrastées dans le temps et l'espace.

Dans des conditions extrêmes de pâturage, les adaptations comportementales mises en ouvre par les animaux trouvent néanmoins leurs limites. Ainsi, les troupeaux d'herbivores domestiques se scindent en sous-groupes en conditions de faibles disponibilités alimentaires. Dans une population de bovins retournés à l'état sauvage, Lazo (1992) a observé que la taille de ces sous-groupes était conditionnée par l'abondance et la distribution de la végétation herbacée. Dudzinski et al (1978) rapportent qu'un troupeau de 330 brebis Mérinos exploitant un secteur de 5000 ha dans l'ouest de l'Australie s'est progressivement scindé en sous-groupes alors que les disponibilités alimentaires se réduisaient. Ces auteurs ont pu mesurer que la dispersion globale du troupeau augmentait alors que l'état corporel des 
Figure 7. Ajustements du comportement d'ingestion et d'exploration de génisses dans des prairies d'estives pâturées en continu dans un gradient de chargement - conséquences sur la dynamique saisonnière du gain de poids vif journalier (d'après Dumont et al 2007).
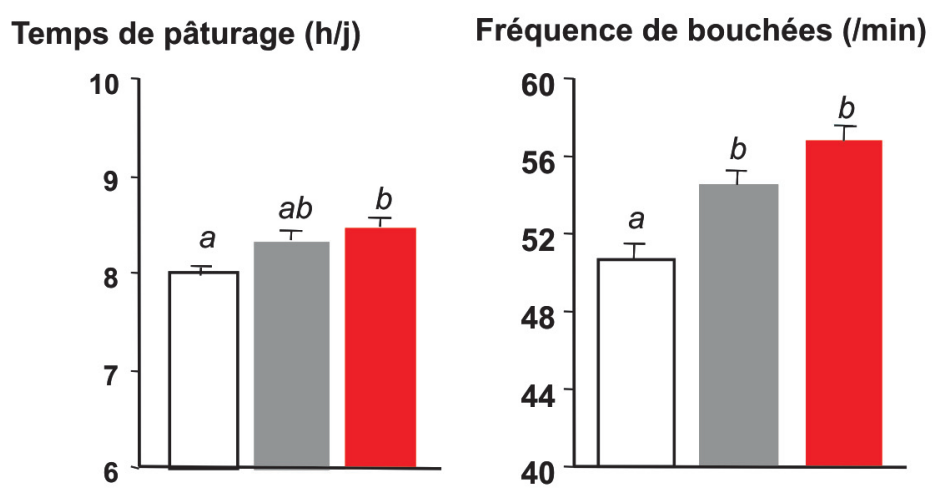

Nombre de bouchées par pas

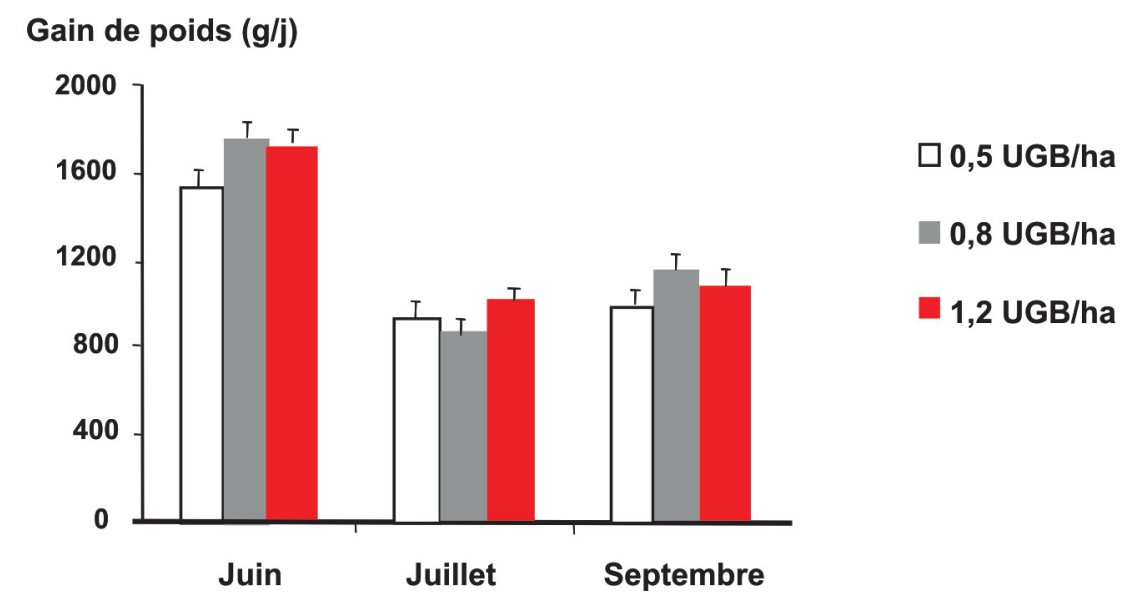

animaux du troupeau diminuait. Au moment le plus critique de la sécheresse, les animaux ont parfois reconstitué des groupes de plus grande taille, traduisant ainsi l'abandon de la quête alimentaire. Des pertes d'animaux ont alors été enregistrées par sous-nutrition, ce qui révèle une situation de rupture.

La flexibilité comportementale des herbivores domestiques s'exprime également lorsque la durée d'accès au pâturage est modulée. Bovins (Chilibroste et al 1997), ovins (Iason et al 1999) et caprins (Romney et al 1996) tentent alors de compenser un temps d'accès au pâturage réduit par une augmentation de leur flux d'ingestion, qui résulte d'une augmentation de la masse de leurs bouchées (Chilibroste et al 1997, Iason et al 1999) et parfois de leur fréquence de préhension (Ginane et Petit 2005). Lorsque les contraintes sont limitées, les animaux arrivent à maintenir leur ingestion journalière par exemple lorsqu'on permet $9 \mathrm{~h} 30$ de pâturage sur un couvert de $5,5 \mathrm{~cm}$ de hauteur pour des ovins (Iason et al 1999), mais en deçà de certains seuils ces ajustements ne suffisent plus. Dans la seule étude où les choix alimentaires étaient également mesurés, une réduction du temps d'accès au pâturage à deux périodes quotidiennes de 2 h 30 chacune a conforté la préférence de génisses pour des repousses végétatives de haute valeur nutritive offertes face à un couvert épié (Ginane et Petit 2005). Cette réaction a été modulée par la hauteur des repousses végétatives, et par le stade de maturité du couvert épié, avec cependant une priorité marquée pour la qualité du régime sélectionné aux dépens des quantités ingérées.

Les expériences durant lesquelles les animaux subissent un jeûne alimentaire permettent également d'apprécier la flexibilité comportementale des herbivores. Après un jeûne de l'ordre de $24 \mathrm{~h}$, ovins (Newman et al 1994) et bovins (Greenwood et Demment 1988) augmentent considérablement leur flux d'ingestion, avec cependant un ajustement différent de leur comportement d'ingestion. Les ovins augmentent leur masse de bouchée, ce qui se traduit dans un premier temps par une diminution de leur fréquence de préhension (Newman et al 1994, Dumont et al 1995), alors que les bovins augmentent d'emblée leur fréquence de préhension (Greenwood et Demment 1988). La différence d'ajustement entre les deux espèces résulte probablement du fait que la contrainte de mastication est moindre pour les bovins, alors que les mouvements de préhension et de mastication sont exclusifs pour les ovins (Dumont et al 1995, Prache et Peyraud 1997). Dans les rares expériences où les préférences alimentaires des animaux étaient également mesurées, les ovins ont augmenté leur consommation relative de ray-grass face au trèfle (Newman et al 1994) et leur consommation d'herbe épiée, ou plus généralement d'un fourrage grossier, face à des repousses végétatives (Dumont et Petit 1995, Dumont et al 1995), alors que les bovins ne modifiaient pas leur préférence relative entre ces deux couverts. Au-delà de ces effets d'une privation alimentaire de courte durée, l'augmentation des besoins des animaux, par exemple durant la phase de lactation, révèle également leur flexibilité comportementale. Comparées à des congénères taries, des vaches (Gibb et al 1999) ou des brebis en lactation (Petit et Béchet 1995) augmentent leur ingestion quotidienne en augmentant leur temps de pâturage journalier et, lorsque la structure du couvert le permet, leur flux d'ingestion. Des vaches en lactation de race Salers (Farruggia et al 2006) ou Charolaises (Farruggia et al 2008) sélectionnent plus les repousses végétatives de haute valeur nutritive que les vaches taries, dans une prairie permanente en fin de saison de pâturage. Un investissement accru des femelles en lactation en direction des 
Figure 8. Deux exemples de comportements qui traduisent la plus grande efficacité de recherche alimentaire de brebis lorsque la distribution du concentré était stable durant les cinq jours de mesure (d'après Hewitson et al 2005). Les bols remplis de trois quantités différentes de concentré sont représentés par différentes nuances de gris.

Bols pleins visités/bois totaux visités

Stable intra- et entre sites : SISE

Jours 1-5

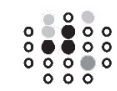

$\therefore: \therefore:$

Variable intra, stable entre sites : VISE

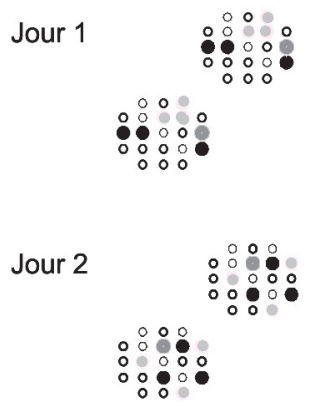

repousses végétatives rases qu'elles préfèrent a d'ailleurs également été observé chez les équidés (Lamoot et al 2005).

Il existe également des mécanismes d'adaptations qui mettent en jeu les capacités cognitives des animaux. Expérimentalement, il a été proposé à des brebis d'exploiter des parcelles de dactyle dans lesquelles la distribution spatiale de sept groupes de bols contenant du concentré (qui n'étaient visibles des animaux qu'à proximité immédiate) était stable ou variable à l'intérieur des sites et entre sites (figure 8, Hewitson et al 2005). Les animaux ont été plus efficaces dans leur recherche lorsque la distribution du concentré était stable durant les cinq jours de mesure, puisqu'ils visitaient alors un nombre proportionnellement plus élevé de bols contenant du concentré (figure 8). Ceci suggère qu'ils ont su utiliser leur mémoire spatiale à l'intérieur même des sites alimentaires alors que classiquement on considérait que celle-ci ne leur servait que pour changer de site d'alimentation (Bailey et al 1996). Ainsi, la réaction comportementale des animaux serait modulée par le niveau auquel se manifeste l'hétérogénéité de leur environnement alimentaire (Hobbs 1999) : ici l'hétérogénéité de chaque site était grande avec des bols vides et des bols contenant jusqu'à $27 \mathrm{~g}$ de concentré, et il s'y exerçait une compétition importante entre les trois animaux des groupes, ce qui rend compte d'une utilisation
Stable intra, variable entre sites : SIVE

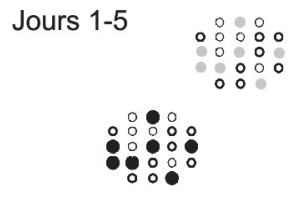

Variable intra- et entre sites : VIVE

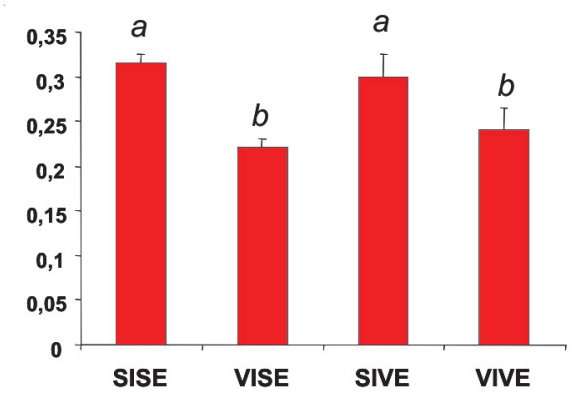

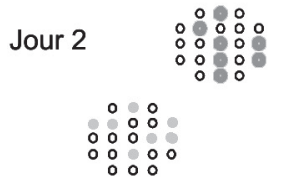

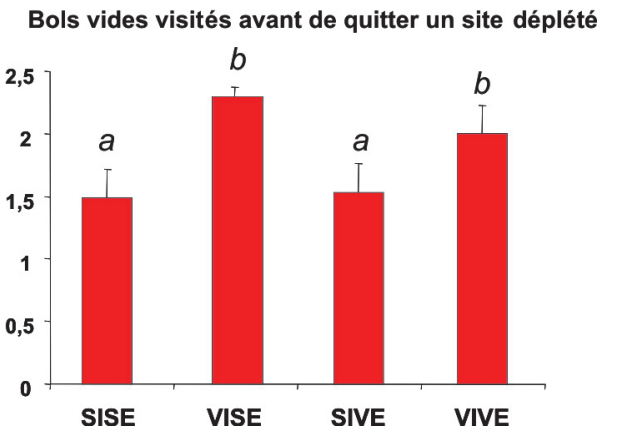

des capacités mémorielles des animaux à une échelle fine. Lorsque la distribution du concentré dans les bols changeait d'un jour à l'autre, les animaux ont en revanche prolongé la recherche du concentré au sein de chaque site alimentaire (figure 8). Ainsi, les animaux semblent avoir partiellement substitué l'utilisation de leur mémoire spatiale par une augmentation de leur comportement exploratoire (Hewitson et al 2005). Dans un autre essai, des agnelles exploitaient un couvert de fétuque dans lequel des placettes de ray-grass étaient soit disséminées, soit réparties en agrégats (Dumont et al 2000). Il a été observé qu'une tactique de déplacements accrus pouvait, dans le cas d'une dissémination des placettes de ray-grass, compenser les bénéfices liés à l'utilisation de la mémoire spatiale lorsque les placettes étaient agrégées. Cette flexibilité comportementale des ovins semble favoriser leur efficacité de recherche alimentaire.

Comme les herbivores domestiques s'alimentent en groupe, ils peuvent bénéficier d'informations détenues par leurs congénères pour leur recherche alimentaire (Bailey et al 1996, Dumont et al 2000), mais peuvent aussi se voir exclure d'une ressource qu'ils ont découverte par des congénères dominants (Appleby 1980). Le rôle de la hiérarchie de dominance a été analysé au moment de la décision de changer de site alimentaire. Hewitson et al (2007) ont pour cela uti-

lisé un test de déplacement dans lequel les animaux pouvaient consommer un foin offert à volonté, mais devaient se déplacer pour obtenir une quantité de concentré distribuée aléatoirement à l'aide d'un dispositif ne permettant qu'à un seul animal de le consommer. Testés seuls, les animaux ont d'autant moins accepté le risque de perdre le foin disponible à volonté que la probabilité d'obtenir le concentré était faible, et ceci indépendamment de leur statut social. Lorsque les animaux étaient testés par paire, les dominants se sont comportés de manière opportuniste, comme prédit pour les «scroungers» (i.e., les «profiteurs») dans les modèles «producersscroungers» (Barnard et Sibly 1981) ; ils ont suivi les subordonnés dans leurs déplacements et les ont empêché de profiter de la récompense obtenue. Les animaux subordonnés ont quant à eux utilisé plus d'information avant de prendre la décision de se déplacer : la probabilité d'obtenir le meilleur aliment, mais aussi leurs expériences antérieures et l'appréciation de l'écart par rapport à leur partenaire dans la hiérarchie de dominance (Hewitson et al 2007). Ainsi la flexibilité comportementale des ovins ne s'exerce-t-elle pas uniquement vis-à-vis de la distribution des ressources au sein de l'environnement dans lequel ils évoluent, mais également vis-à-vis de la présence de leurs congénères.

Dans les exemples précédemment décrits, il a été question d'élasticité 
puisque une fois la contrainte supprimée les animaux retrouvaient leur comportement initial. Dans le cas des apprentissages alimentaires, on peut cependant parler de plasticité comportementale parce que le comportement des animaux est modifié dans la durée. Chez le jeune, les processus d'apprentissage par imitation sont à la base de la transmission sociale des préférences alimentaires. Ainsi des agneaux en contact avec une brebis en train de consommer un aliment qui leur est inconnu, l'acceptent par la suite plus volontiers que des agneaux qui avaient été exposés seuls à cet aliment (Thorhallsdottir et al 1990). De même, en situation de choix, la préférence pour un aliment persiste plus longtemps chez des agneaux exposés préalablement à cet aliment avec leur mère que chez des agneaux exposés seuls (Nolte et al 1990). Au pâturage, la transmission sociale des préférences alimentaires permet aux jeunes animaux et aux animaux récemment introduits dans un nouvel environnement d'exploiter efficacement le milieu qu'ils découvrent. Ceci a été aussi bien observé sur des associations simples entre graminées et légumineuses (Orr et al 1995) que sur des parcours diversifiés en zone méditerranéenne (Lécrivain et al 1996) ou tropicale (Biquand et BiquandGuyot 1992).

La période au cours de laquelle l'appareil digestif $\mathrm{du}$ jeune ruminant se transforme semble propice à une imprégnation alimentaire (Squibb et al 1990). Ainsi, aux processus d'apprentissage, s'ajoute probablement une adaptation physiologique des animaux aux fourrages précocement consommés. Ce serait, par exemple, en partie du fait d'un meilleur recyclage de l'azote que des agneaux qui ont consommé autour du sevrage des fourrages riches en fibres et de faible valeur nutritive, en ingèrent plus neuf mois plus tard que des animaux «naïfs» (Distel et al 1996). De même, des génisses laitières qui ont appris à pâturer au cours de leur première année ont une plus forte reprise de poids et une meilleure fertilité en première lactation que des animaux élevés à l'étable avec une alimentation à base de fourrages conservés. En plus du processus d'apprentissage, l'adaptation physiologique des animaux explique leur plus forte consommation d'herbe : les génisses ayant été au pâturage sembleraient avoir une capacité d'ingestion plus élevée en fin de gestation et au début de la première lactation qui résulterait pour partie d'un développement plus important de leur rumen (Troccon 1993).

Il ressort ainsi que les réponses adaptatives des herbivores se construisent à partir d'une combinaison de processus physiologiques et comportementaux. Dans la partie suivante l'analyse de ces réponses s'enrichit d'une comparaison entre races dans des situations où les animaux sont soumis à une même contrainte. Cette approche permet de s'interroger sur l'efficacité des stratégies adaptatives mises en œuvre par les différents génotypes pour répondre à des priorités «collectives» (assurer la pérennité du troupeau, optimiser sa productivité) vs «individuelles» (assurer sa propre survie et sa longévité) dans un environnement fluctuant.

\section{4 / Variabilité des trajectoi- res adaptatives et avantages adaptatifs comparés}

Les comparaisons entre races permettent dans un premier temps d'explorer la variabilité des réponses entre génotypes et dans un second temps de s'appuyer sur cette variabilité pour proposer des interprétations en termes d'avantages adaptatifs. C'est le cas par exemple du suivi comparatif portant sur la sélection alimentaire dans des prairies naturelles de moyenne montagne, de vaches taries et en lactation (Farruggia et al 2006, 2008). Dans cette étude, les vaches en lactation, qu'elles soient de race Charolaise ou Salers, ont continué à sélectionner les repousses végétatives jusqu'en toute fin de saison, et cela malgré leur très faible hauteur. En fin de lactation, il a cependant été observé une allocation différente des nutriments ainsi sélectionnés entre ces deux races. Chez les Salers, on observe une corrélation positive entre la sélection des repousses végétatives et le niveau de production laitière, sans que les réserves corporelles ne soient mises à contribution (Farruggia et al 2006). Chez les Charolaises, au moindre potentiel laitier, il n'a pas été observé de corrélation entre la sélection des repousses végétatives et le niveau de production laitière des animaux. En revanche, les vaches qui sélectionnaient plus les repousses végétatives ont perdu moins de poids en fin de saison (Farruggia et al 2008). Ainsi, la flexibilité comportementale des vaches en lactation qui se traduit par une augmentation de leur sélectivité alimentaire a eu des effets différents sur les performances animales selon des orientations métaboliques propres à chacune des deux races.

Des différences de réponses entre races peuvent également être appréciées par des approches plus prédictives de modélisation statistique et de simulation. Celles-ci ont été mobilisées pour comparer les productivités de plusieurs races bovines dans une même gamme de niveaux alimentaires simulés (Jenkins et Ferrell 1992, 1996) ou en réponse à différents systèmes d'alimentation (Bryant et al 2006). Cette démarche est intéressante car elle permet de décrire les courbes de réponses de chaque indicateur de performance étudié (productivité numérique, croissance et survie du veau, poids vif des vaches, production laitière, qualité du lait), et de comparer ainsi leur sensibilité à la variation du niveau des apports alimentaires. Il devient alors possible d'estimer la contribution de chacun de ces indicateurs à la construction d'une performance globale (efficacité de production) selon les niveaux alimentaires ou les systèmes d'alimentation, et d'analyser, dans un second temps, les différences d'efficacité entre races (figure 9, Jenkins et Ferrell 1994). Ainsi en système extensif où l'ingestion journalière peut être temporairement limitée, les animaux de race Red Poll et Angus semblent être plus efficaces pour produire des veaux (reproduction et

Figure 9. Evolution de l'efficacité biologique prédite ( $g$ de veau sevré par kg de MS ingéré par sa mère) selon le niveau d'ingestion de vaches appartenant à 4 races différentes (d'après Jenkins et Ferrell 1994).

\section{Efficacité biologique}

(g de veau sevré/kg MS ingéré)

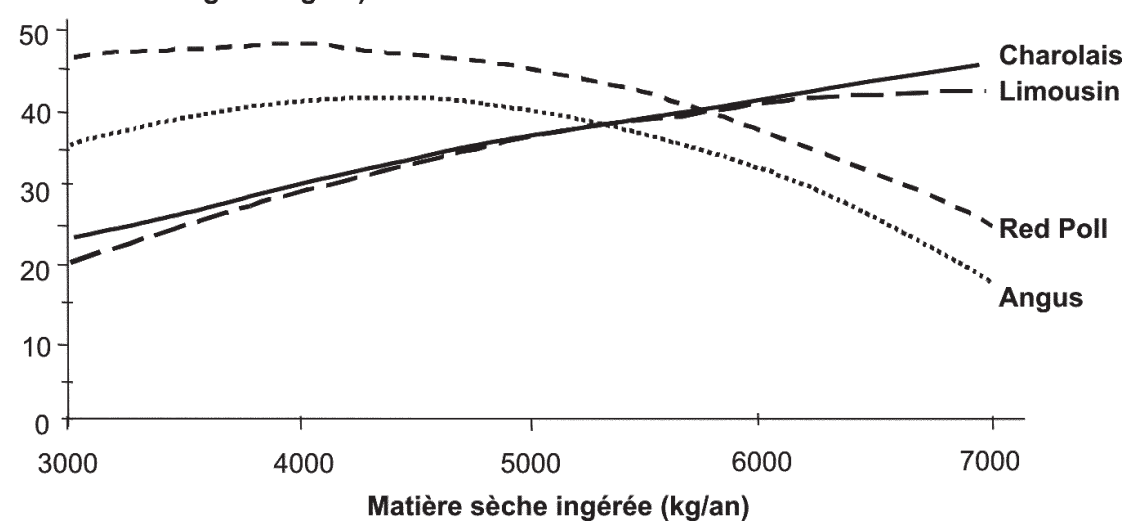


croissance) que les vaches Charolaises et Limousines. En revanche dès que les conditions alimentaires deviennent plus favorables, ces dernières mettent davantage à profit les potentialités du milieu et expriment une efficacité biologique supérieure. Concernant les races laitières, Bryant et al (2006) soulignent que dans des environnements hétérogènes, cette efficacité est plus élevée pour les génotypes qualifiés de généralistes (New Zealand Friesan, Jersiaise) que pour les génotypes spécialistes (Hosltein).

Les différences de stratégies adaptatives observées entre races trouvent un intérêt à être étudiées sur le long terme et en considérant plusieurs critères pertinents (comportementaux et zootechniques) afin de pouvoir mieux apprécier l'efficacité de ces stratégies non seulement du point de vue de la productivité (fitness «zootechnique») mais aussi de l'aptitude des femelles à se reproduire (fitness «biologique», transmission des gènes) et à survivre dans cet environnement (longévité). Une telle approche «multi-traits» a été proposée par D'hour et al (1995). Un suivi comparatif entre vaches de races Salers et Limousine a ainsi été mené depuis le sevrage jusqu'à la quatrième lactation, afin d'enregistrer les trajectoires zootechniques de vaches Salers et Limousine placées dans des systèmes de conduite identiques et soumises à deux niveaux d'alimentation contrastés (Haut vs Bas). Ces différences de niveaux alimentaires se sont opérées chaque année de la mi-août jusqu'à fin avril, en jouant tout d'abord sur le chargement au pâturage, puis sur les niveaux d'alimentation hivernale. Les vaches appartenant aux lots Haut ont été nourries selon leurs besoins, tandis que les besoins énergétiques des vaches des lots Bas n'étaient couverts qu'à hauteur de $80 \%$. Seule une période d'alimentation libérale au pâturage, d'une durée de 3,5 mois, était commune chaque année à tous les animaux.

La sous-alimentation hivernale n'a pas affecté la production laitière moyenne des vaches Salers (- 0,5 kg, NS), traduisant ainsi une certaine résistance de la fonction de lactation à une sousalimentation modérée chez cette race. En revanche, la réponse des Limousines a été flexible puisque la sous-alimentation hivernale a fait chuter la production laitière en moyenne de - 1 à - $1,5 \mathrm{~kg}$ de lait/j $(\mathrm{p}<0,01)$ (figure 10a). Après la mise à l'herbe, l'évolution des productions laitières des vaches des lots Bas révèle une flexibilité chez les deux races mais qui se caractérise par des amplitudes différentes. Ainsi, chez les Salers, la production laitière des vaches du lot Bas dépasse celle des vaches du lot Haut tandis que chez les Limousines la production laitière des vaches du lot Bas parvient uniquement à rattraper celle des vaches du lot bien alimenté (figure 10a). Cette différence de réponse entre les deux races peut s'interpréter par des réserves adipeuses relativement plus importantes (maturité atteinte) et plus facilement mobilisables chez les Salers, par des différences d'ingestion au pâturage, ou encore par des différences d'orientations métaboliques entre races. Cette dernière hypothèse est d'ailleurs confortée par l'analyse des évolutions de poids vif des vaches. Alors que chez les Salers, l'écart de poids vif de $50 \mathrm{~kg}$ observé au premier vêlage entre les 2 niveaux alimentaires est maintenu à l'issue de 4 lactations, le même écart initial s'est totalement estompé chez les Limousines, sans que l'on observe de dérive liée à la politique de réforme (poids vifs des vaches de réforme des deux races identiques à ceux des vaches conservées). Ainsi, il semblerait que chez la vache Salers la production de lait destinée à la croissance de son veau soit prioritaire, tandis que chez la Limousine la priorité porte davantage sur le développement corporel. En ce qui concerne l'aptitude à se reproduire, le niveau alimentaire Bas a eu pour effet d'induire un délai de reprise de cyclicité après le vêlage dans les deux races. Ce délai a toutefois été presque deux fois plus important chez les Limousines $(+12 \mathrm{j})$ que chez les Salers $(+7 \mathrm{j})$ (D’hour et Petit 1997). Sur le long terme, après quatre lactations, des différences de trajectoires adaptatives entre races se dessinent. Celles-ci sont révélées par des mises sous contraintes (ressources alimentaires limitantes) et ont des conséquences sur le taux de survie des vaches. Dans une situation où la politique de réforme concerne presque exclusivement les échecs de la

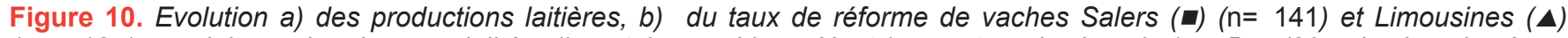
$(\mathrm{n}=127)$ conduites selon deux modalités alimentaires en hiver: Haut (couverture des besoins) vs Bas (80\% des besoins énergétiques couverts en hiver) durant quatre années successives. (d'après D'hour et Petit 1997, D'hour et al 1995).

\section{a) Production laitiére $(\mathrm{kg} / \mathrm{j})$}

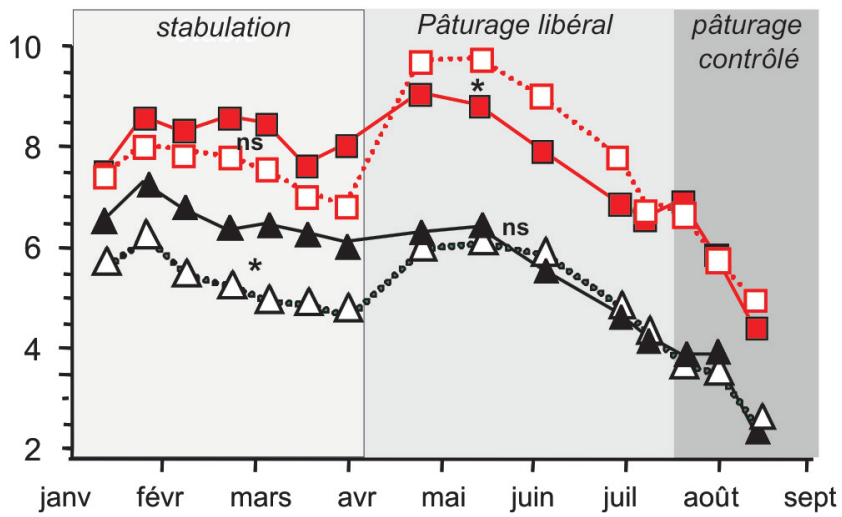

b) Taux de réforme des vaches (\% de l'effectif initial)

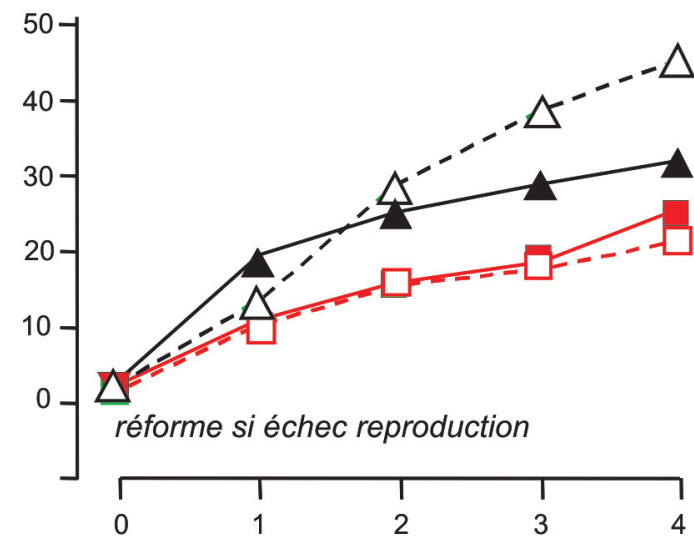

Niveau alimentaire

Parité

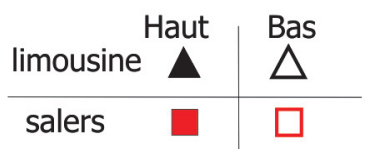

*, différence significative $\mathrm{P}<0,05$ entre les productions laitières des niveaux Haut et Bas. 
reproduction, l'évolution du pourcentage de vaches restant dans le troupeau d'une année sur l'autre rend compte de l'aptitude des femelles à être gravide dans les délais imposés par l'éleveur. Sur ce critère également des différences nettes apparaissent entre les deux races. Globalement les vaches Limousines ont une moindre aptitude à se reproduire par rapport aux vaches Salers (taux de vaches vides plus important en fin de saison de reproduction). Dans le cas de conduites où les femelles sont soumises à des périodes de sous-alimentations hivernales, l'aptitude des femelles Salers à se reproduire se maintient (résistance) proche de celle des vaches du lot bien alimenté. $\mathrm{Au}$ contraire, les vaches Limousines du lot Bas ont une aptitude à la reproduction qui se dégrade d'une année sur l'autre : l'écart entre le taux de réforme des vaches Limousine du lot Haut et celui des Limousine du lot Bas s'amplifie au cours du temps (figure $10 b)$. Ces résultats révèlent la moindre aptitude des vaches Limousines à se reproduire dès lors qu'elles sont placées en situation de contrainte alimentaire. Ils mettent également en évidence chez cette race, des effets sur la survie des femelles d'autant plus marqués que la contrainte est répétée dans le temps et qu'elle s'accompagne d'une politique de réforme stricte sur les individus ne parvenant pas à initier une gestation dans les délais imposés par l'éleveur. Du fait de la politique de réforme retenue, la stratégie adaptative des vaches Limousines apparaît globalement moins efficace que celle observée chez les vaches Salers en situation de contrainte alimentaire. Ce constat peut être réalisé aussi bien à l'échelle du troupeau (mise en péril de la pérennité du troupeau du fait de la dégradation des performances de reproduction) qu'à celle de l'individu (risque de réforme accru et moindre longévité). Ainsi, dans les systèmes d'élevage extensifs où les ressources alimentaires sont fluctuantes et peuvent être temporairement limitantes au moment de la période de reproduction, certains génotypes développent des stratégies adaptatives plus efficaces que d'autres. Cette efficacité peut être considérablement amplifiée dans les systèmes pilotés dès lors que les décisions de réforme pénalisent systématiquement et sans compromis les femelles vides à l'issue de la période de reproduction.
A l'échelle de sa carrière, la survie d'un individu intègre non seulement sa capacité à faire face aux contraintes de l'environnement mais aussi à répondre aux contraintes imposées par l'éleveur. Analyser l'efficacité des réponses adaptatives des femelles reproductrices à cette échelle, nécessite de développer une approche combinant des traits caractérisant la performance individuelle et des traits caractérisant l'aptitude de l'animal à se reproduire dans une gamme variable de conditions nutritionnelles. Il devient alors intéressant de se poser la question de l'adaptabilité des animaux, mais aussi des effets de la sélection sur celle-ci, en considérant la façon dont les priorités s'orientent, au cours du cycle de production et à l'échelle de la carrière, respectivement vers les fonctions de survie, de production et de reproduction (Friggens et al 2010).

Sur le plan pratique, la variabilité des trajectoires adaptatives observée entre individus et entre génotypes peut être appréciée comme un levier potentiel pour l'éleveur pour gérer l'aléa. Lorsque la situation économique de l'exploitation le permet, les éleveurs ont tendance à gérer une pénurie alimentaire (sécheresse) en ayant recours à l'achat d'aliments qui leur permet de maintenir les objectifs de production du troupeau. Ils ont, de ce point de vue, une attitude averse au risque assez marquée (Mosnier 2009). En revanche, dans les systèmes très extensifs, ne bénéficiant pas d'aides, les achats d'aliments ne sont pas toujours possibles. Ce sont alors les animaux qui supportent les fluctuations de la ressource fourragère disponible. De ce fait, la gestion de l'aléa climatique repose principalement sur leur capacité à mobiliser et à reconstituer leurs réserves corporelles. Un tel levier est ainsi activé dans les systèmes pastoraux de la puna andine, de l'Atlas marocain ou de la steppe mongole où les éleveurs acceptent alors d'avoir des animaux temporairement amaigris (Brunschwig et al 2007). La flexibilité des animaux peut ainsi être considérée comme un élément de sécurisation du système et un levier à mobiliser de façon raisonnée et progressive selon l'intensité et le caractère aléatoire des contraintes subies.

\section{Conclusion}

Les systèmes d'élevage extensifs de ruminants sont particuliers car le milieu dans lequel évoluent les animaux est difficilement contrôlable par l'Homme. $\mathrm{Ce}$ milieu est de fait très hétérogène et fluctuant. Cette revue bibliographique révèle comment les animaux sont capables de s'adapter individuellement aux contraintes de tels milieux, et ceci avec une grande variabilité dans les modalités de réponses (physiologiques et métaboliques et/ou comportementales) et les niveaux de réponse (sensibilité). Cette variabilité individuelle contribue à faire émerger à l'échelle du troupeau des propriétés de robustesse. Sur le court et le moyen terme, celle-ci résulte de phénomènes de compensation entre les réponses des individus. Elle permet globalement à la composante animale du système d'être pérenne, c'est-à-dire de se reproduire tout en assurant un minimum de production. Cette robustesse apparaît d'autant plus nettement que le troupeau est de taille importante. Sur le long terme, la variabilité individuelle est susceptible de générer de la robustesse en rendant possible une sélection génétique pour les caractères adaptatifs les plus pertinents dans un contexte d'élevage donné (Simm et al 1996).

Un autre intérêt de la flexibilité des animaux est de permettre à l'éleveur d'adopter des conduites différentes vis-à-vis de la gestion de l'aléa climatique auquel les systèmes extensifs sont particulièrement sensibles et auxquels ils risquent d'être de plus en plus confrontés si l'on se réfère aux perspectives d'évolutions du climat dans le contexte de réchauffement global.

\section{Remerciements}

Les auteurs tiennent à remercier les personnels des unités expérimentales de l'INRA, Unité Expérimentale des Monts d'Auvergne, Unité Expérimentale du Pin au Haras, Installation expérimentale de Fréjorgues, qui ont largement pris part aux études mobilisées dans cette synthèse. 


\section{Références}

Agreil C., Fritz H., Meuret M., 2005. Maintenance of daily intake through bite mass diversity adjustment in sheep grazing on heterogeneous and variable vegetation. Appl. Anim. Behav. Sci., 91, 35-56.

Alcaras J.R., Lacroux F., 1999. Planifier, c'est s'adapter. Economies et Sociétés, Sciences de Gestion, Série S.G., 26-27 (6-7), 7-37.

Allden W.G., Whitaker I.A.McD., 1970. The determinants of herbage intake by grazing sheep: The interrelationship of factors influencing herbage intake and availability. Aust. J. Agric. Res., 21, 755-766.

Appleby M.C., 1980. Social rank and food access in red deer stags. Behaviour, 74, 294 309.

Atti N., Bocquier F., 1999. Adaptation des brebis Barbarine à l'alternance sous-nutritionréalimentation : effets sur les tissus adipeux. Ann. Zootech., 48, 189-198.

Bailey D.W., Gross J.E., Laca E.A., Rittenhouse L.R., Coughenour M.B., Swift D.M., Sims P.L., 1996. Mechanisms that result in large herbivore grazing distribution patterns. J. Range Manag., 49, 386-400.

Barnard C.J., Sibly R.M., 1981. Producers and scroungers: a general model and its application to captive flocks of house sparrows. Anim. Behav., 29, 543-550.

Biquand S., Biquand-Guyot V., 1992. The influence of peers, lineage and environment on food selection of the criollo goat (Capra hircus). Appl. Anim. Behav. Sci., 34, 231-245.

Blanc F., Agabriel J., 2008. Modelling the reproductive efficiency in a beef cow herd: effect of calving date, bull exposure and body condition at calving on the calving-conception interval and calving distribution. J. Agric. Sci., 146, 143-161.

Blanc F., Bocquier F., Agabriel J., D'hour P., Chilliard Y., 2004. Amélioration de l'autonomie alimentaire des élevages de ruminants : conséquences sur les fonctions de production et la longévité des femelles. Renc. Rech. Rum., 11, 155-162.

Blanc F., Bocquier F., Agabriel J., D'Hour P., Chilliard Y., 2006. Adaptive abilities of the females and sustainability of ruminant livestock systems: a review. Anim. Res., 55, 489510 .

Blanc F., Fabre D., Bocquier F., Canepa S., Delavaud C., Caraty A., Chilliard Y., Debus N., 2007. Effect of a post-weaning restricted nutrition on the initiation of puberty and the reproductive performances of early bred Merino ewe-lambs. In: Advanced nutrition and feeding strategies to improve sheep and goat production. Proc. $11^{\text {th }}$ Sem. FAO-CIHEAM SubNetwork on Sheep and Goat Nutrition. Priolo A., Biondi L., Ben Salem H., Morand-Fehr P. (Eds). Opt. Méd., Série A, 74, 387-393.

Bocquier F., Leboeuf B., Rouel J., Chilliard Y., 1998. Effet de l'alimentation et des facteurs d'élevage sur les performances de reproduction des chevrettes Alpines. INRA Prod. Anim., 11, 311-320.

Boval M., Fanchone A, Archimède $\mathrm{H}$, Gibb M.J., 2007. Effect of structure of a tropical pasture on ingestive behaviour, digestibility of diet and daily intake by grazing cattle. Grass Forage Sci., 62, 44-54.
Brunschwig G., 1992. La haute vallée du Cañete : la lande et la puna. In : Comprendre l'agriculture paysanne dans les Andes Centrales, Morlon P. (Eds). INRA, Paris, France, 383-409.

Brunschwig G., Devienne S., Bourbouze A., 2007. Regards croisés sur la gestion des élevages pastoraux en conditions de milieu difficiles: analyse de cas dans les Andes centrales péruviennes, la steppe mongole et l'Atlas marocain. Renc. Rech. Rum., 14,195.

Bryant J.R., Lopez-Villalobos N., Pryce, J.E., Holmes, C.W., Johnson D.L., 2006 Reaction norms used to quantify the responses of New Zealand dairy cattle of mixed breeds to nutritional environment. N. Z. J. Agric. Res., $49,371-381$

Butler W.R., 2003. Energy balance relationships with follicular development ovulation and fertility in postpartum dairy cows. Livest. Prod. Sci., 83, 211-218.

Chilibroste P., Tamminga S., Boer H., 1997. Effects of length of grazing session, rumen fil and starvation time before grazing on dry-matter intake, ingestive behaviour and dry-matter rumen pool sizes of grazing lactating dairy cows. Grass Forage Sci., 52, 249-257.

Chilliard Y., Bocquier F., Doreau M., 1998a. Digestive and metabolic adaptations of ruminants to undernutrition, and consequences on reproduction. Reprod. Nutr. Dev., 38, 131-152.

Chilliard Y., Doreau M., Bocquier F., 1998b. Les adaptations à la sous-nutrition chez les herbivores. Cahiers de Nutrition et de Diététique, $33,217-224$.

Chilliard Y, Ferlay A, Faulconnier Y, Bonnet M Rouel J, Bocquier F., 2000. Adipose tissue metabolism and its role in adaptations to undernutrition in ruminants. Proc. Nutr. Soc., 59, 127-34.

Debayle J., 2004. Une exploitation ovine pastorale de Provence appelée à gérer la biodiversité. Fourrages, 179, 447-449.

Dedieu B., Chai E., Leclerc B., Moulin C.H., Tichit M., 2008. L'élevage en mouvement flexibilité et adaptation des exploitations d'herbivores. Dedieu B., Chia E., Leclerc B. Moulin C.H., Tichit M. (Eds). Editions Quae, Paris, France, 296p.

D'hour P., Petit M., Pradel P., Garel J.P., 1995. Evolution du poids et de la production laitière au pâturage de vaches salers et limousines dans deux milieux. Renc. Rech. Rum., 2 105-108.

D'hour P., Petit M., 1997. Influence of nutritional environment on reproductive performances of Limousin and Salers cows. In: Suckle cow workers meeting, Kirbymoorside. Pullar (Ed). Proc. UK, 15-18

Diskin M.G., Mackey D.R., Roche F. Sreenan J.M., 2003. Effects of nutrition and metabolic status on circulating hormones and ovarian follicle development in cattle. Anim. Reprod. Sci., 78, 345-370.

Distel R.A., Villalba J.J., Laborde H.E., Burgos M.A., 1996. Persistence of the effects of early experience on consumption of lowquality roughage by sheep. J. Anim. Sci., 74, 965-968.

Dozias D., Agabriel J., Pecatte J.R., Petit M., 2006. Influence du profil de croissance des génisses des troupeaux allaitants sur leurs performances ultérieures. Actes des Journées Nationales des GTV, 145-154.

Dudzinski M.L., Schuh H.J., Wilcox D.G., Gardiner H.G., Morrissey J.G., 1978. Statistical and probabilistic estimators of forage conditions from grazing behaviour of Merino sheep in a semi-arid environment, Appl. Anim. Ethol., 4, 357-368.

Dumont B., Petit M., 1995. An indoor method for studying the preferences of sheep and cattle at pasture. Appl. Anim. Behav. Sci., 46, 67-80.

Dumont B., Petit M., D'hour P., 1995 Choice of sheep and cattle between vegetative and reproductive cocksfoot patches. Appl. Anim. Behav. Sci., 43, 1-15.

Dumont B., Maillard J.F., Petit M., 2000. The effect of the spatial distribution of plant species within the sward on the searching success of sheep when grazing. Grass Forage Sci., $55,138-145$

Dumont B., Meuret M., Boissy A., Petit M., 2001. Le pâturage vu par l'animal : mécanismes comportementaux et applications en élevage. Fourrages, 166, 213-238.

Dumont B., Garel J.P., Ginane C., Decuq F., Farruggia A., Pradel P., Rigolot C., Petit M., 2007. Effect of cattle grazing a species-rich mountain pasture under different stocking rates on the dynamics of diet selection and sward structure. Animal, 1, 1042-1052.

Farruggia A., Dumont B., D'hour P., Egal D., Petit M., 2006. Diet selection of dry and lactating beef cows grazing extensive pastures in late autumn. Grass Forage Sci., 61, 347-353.

Farruggia A., Dumont B., D'hour P., Egal D., 2008. How does protein supplementation affect the selectivity and performance of Charolais cows on extensively grazed pastures in late autumn? Grass Forage Sci., 63, 314-323.

Ferrer Cazcarra R., Petit M., 1995. The effect of winter feeding level on subsequent grazing behavior and herbage intake of Charolais heifers. Anim. Sci., 61, 211-217.

Fleurance G., Dumont B., Farruggia A., Edouard N., Lanore L., 2009. Effect of grazing intensity on foraging behaviour and patch selection by horses. Proc. Int. Ethological Conf., Rennes, France, August 19-24th, abstract.

Foster D.L., Yellon S.M., Olster D.H., 1985. Internal and external determinants of the timing of puberty in the female. J. Reprod. Fert., 75, 327-344

Friggens N.C., 2003. Body lipid reserves and the reproductive cycle: towards a better understanding. Livest. Prod. Sci., 83, 219-236.

Friggens N.C., Disenhaus C., Petit H.V., 2010. Nutritional sub-fertility in the dairy cow: towards improved reproductive management through a better biological understanding. Animal, accepté pour publication, doi:10.1017/S1751731109991601

Garcia F., Carrère P., Soussana J.F., Baumont R., 2003. The ability of sheep at different stocking rates to maintain the quality and quantity of their diet during the grazing season. J. Agric. Sci., 140, 113-124. 
Gibb M.J., Huckle C.A., Nuthall R., Rook A.J., 1999. The effect of physiological state (lactating or dry) and sward surface height on grazing behaviour and intake by dairy cows. Appl. Anim. Behav. Sci., 63, 269-287.

Ginane C., Petit M., 2005. Constraining the time available to graze reinforces heifers' preference for sward of high quality despite low availability. Appl. Anim. Behav. Sci., 94, 1-14.

Greenwood G.B., Demment M.W., 1988. The effect of fasting on short-term cattle grazing behaviour. Grass Forage Sci., 43, 377-386.

Hewitson L., Dumont B., Gordon I.J., 2005. Response of foraging sheep to variability in the spatial distribution of resources. Anim. Behav., 69, 1069-1076.

Hewitson L., Gordon I.J., Dumont B., 2007. Social context affects patch-leaving decisions of sheep in a variable environment. Anim. Behav., 74, 239-246.

Hobbs N.T., 1999. Response of large herbivores to spatial heterogeneity in ecosystems. In: Nutritional Ecology of Herbivores. Jung H.J.G., Fahey Jr. G.C. (Eds). Proc. Vth Int. Symp. Nutr. Herbivores, San Antonio, USA, 97-129.

Hoch T., Begon C., Cassar-Malek I., Picard B., Savary-Auzeloux I., 2003. Mécanimes et conséquences de la croissance compensatrice chez les ruminants. INRA Prod. Anim., 16, 49-59.

Hoch T., Jurie C., Pradel P., Cassar-Malek I., Jailler R., Picard B., Agabriel J., 2005. Effects of hay quality on intake, growth path, body composition and muscle characteristics of Salers heifers. Anim. Res., 54, 241-257.

Iason G.R., Mantecon A.R., Sim D.A., Gonzalez J., Foreman E., Bermudez F.F., Elston D.A., 1999. Can grazing sheep compensate for a daily foraging time constraint? J. Anim. Ecol., 68, 87-93.

INRA, 2007. Alimentation des bovins, ovins et caprins. Besoins des animaux. Valeurs des aliments, Tables INRA 2007. Editions Quae, Paris, France, 307p.

Isselstein J., Griffith B.A., Pradel P., Venerus S., 2007. Effects of livestock breed and grazing intensity on biodiversity and production in grazing systems. 1 . Nutritive value of herbage and livestock performance. Grass Forage Sci., $62,145-158$

Jenkins T.G., Ferrell C.L., 1994. Productivity through weaning of nine breeds of cattle under varying feed availabilities: I. Initial evaluation. J. Anim. Sci., 72, 2787-2797.

Landais E., Balent G., 1995. Introduction à l'étude des pratiques d'élevage extensif. In : Pratiques d'élevage extensif. Identifier, modéliser, évaluer. Landais E., Balent G. (Eds). Etudes et Recherches sur les Systèmes Agraires et le Développement. INRA Editions, Versailles, France, 13-35.
Lamoot I., Vandenberghe C., Bauwens D., Hoffman M., 2005. Grazing behaviour of freeranging donkeys and Shetland ponies in different reproductive states. J. Ethol., 23, 19-27.

Lazo A., 1992. Facteurs déterminants du comportement grégaire de bovines retournés à l'état sauvage. Rev. Ecol., Terre Vie, 47, 51-66.

Lécrivain E., Abreu da Silva M., Demarquet F., Lasseur J., 1996. Influence du mode d'élevage des agnelles de renouvellement sur leur comportement au pâturage et leurs performances zootechniques. Renc. Rech. Rum., 3, 249-252.

Le Cozler Y., Lollivier V., Lacasse P., Disenhaus C., 2008 Rearing strategy and optimizing first-calving targets in dairy heifers: a review. Animal, 2, 1393-1404.

Mackey D.R., Wylie, A.R.G., Sreenan J.M., Roche, J.F., Diskin M.G., 2000. The effect of acute nutritional change on follicle wave turnover, gonadotropin, and steroid concentration in beef heifers. J. Anim. Sci., 78, 429-442.

Meuret M., 1993. Piloter l'ingestion au pâturage. Et. Rech. Syst. Agric. Dev., 27, 161-198.

Mosnier C., 2009, Adaptation des élevages de bovins allaitants aux aléas de prix et de climat : approches par modélisation. Thèse de doctorat AgroParisTech., Paris, France, 285p.

Mayer A.C., Stöckli V., Huovinen C., Konold W., Estermann B.L., Kreuzer M., 2003. Herbage selection by cattle on sub-alpine wood pastures. Forage Ecol. Manag., 181, 39-50.

Monget P., Chabrolle C., Dupont J. 2008. Tissu adipeux, nutrition et reproduction : quel lien? Bull. Acad. Nat. Méd., 192, 637-648.

Newman J.A., Penning P.D., Parsons A.J., Harvey A., Orr R.J., 1994. Fasting affects intake behaviour and diet preference of grazing sheep. Anim. Behav., 47, 185-193.

Nolte D.L., Provenza F.D., Balph D.F., 1990. The establishment and persistence of food preferences in lambs exposed to selected foods. J. Anim. Sci., 68, 998-1002.

Orr R.J., Penning P.D., Parsons A.J., Harvey A., Newman J.A., 1995. The role of learning experience in the development of dietary choice by sheep and goats. Ann. Zootch., 44, Suppl, 111.

Ortega I.M., Soltero-Gardea S., Drawe D.L., Bryant F.C., 1997. Evaluating grazing strategies for cattle: nutrition of cattle and deer. $J$. Range Manag., 50, 631-637.

Penning P.D., Parsons A.J., Orr R.J., Hooper G.E., 1991. Intake and behaviour responses by sheep to changes in sward characteristics under continuous stocking. Grass Forage Sci., 46, 1528.

Penning P.D., Parsons A.J., Orr R.J., Hooper G.E., 1994. Intake and behaviour responses by sheep to changes in sward characteristics under rotational grazing. Grass Forage Sci., 49, 476486.
Petit M., Béchet G., 1995. Grass intake and grazing behaviour of dry and suckling ewes according to sward height. Ann. Zootech., 44, Suppl, 250.

Petit M., Jarrige R., Russel A.J.F., Wright I.A. 1992. Feeding and nutrition of the suckler cow. In: Beef cattle production. Béranger $\mathrm{C}$. (Ed). World Animal Science, C5, 488p.

Prache S., Peyraud J.L., 1997. Préhensibilité de l'herbe pâturée chez les bovins et les ovins. INRA Prod. Anim., 10, 377-390.

Richards M.W., Spitzer J.C., Warner M.B., 1986. Effect of varying levels of postpartum nutrition and body condition at calving on subsequent reproductive performance in beef cattle. J. Anim. Sci., 62, 300-306.

Robinson J.J., Ashworth C.J., Rooke J.A., Mitchell L.M., McEvoy T.G., 2006. Nutrition and fertility in ruminant livestock. Anim. Feed Sci. Technol., 126, 259-276.

Romney D.L., Sendalo D.S.C., Owen E., Mtenga L.A., Penning P.D., Mayes R.W., Hendy C.R.C., 1996. Effects of tethering management on feed intake and behaviour of Tanzanian goats. Small Rum. Res., 19, 113120.

Sainz R.D., De La Torre F., Oltjen J.W., 1995. Compensatory growth and carcass quality in growth-restricted and reefed beef steers. J. Anim. Sci. 73, 2971-2979.

Schlegel M.L., Wachenheim C.J., Benson M.E., Ames N.K., Rust S.R., 2000. Grazing methods and stocking rates for direct-seeded alfalfa pastures: II. Pasture quality and diet selection. J. Anim. Sci., 78, 2202-2208.

Simm G., Conington J., Bishop S.C., Dwyer C.M., Pattinson S., 1996. Genetic selection for extensive conditions. Appl. Anim. Behav. Sci., 49, 47-59.

Squibb R.C., Provenza F.D., Balph D.F., 1990. Effect of age of exposure on consumption of a shrub by sheep. J. Anim. Sci., 68, 987997.

Suttie J.M., Foster D.L., Veenvliet T.R., Manley T.R., Corson I.D., 1991. Influence of food intake but independence of body weight on puberty in female sheep. J. Reprod. Fert., 92, 33-39.

Thorhallsdottir A.G., Provenza F.D., Balph D.F., 1990. Ability of lambs to learn about novel food while observing or participating with social models. Appl. Anim. Behav. Sci., $25,25-33$.

Tolkamp B.J., Emmans G.C., Kyriazakis I., 2006. Body fatness affects feed intake of sheep at a given body weight. J. Anim. Sci., 84, 17781789 .

Troccon J.L., 1993. Elevage des génisses laitières avec ou sans pâturage. Ann. Zootech., 42, 271-288. 


\title{
Résumé
}

Les systèmes d'élevage extensifs de ruminants reposent largement sur l'exploitation de l'herbe. Leur objectif est de produire tout en maintenant des coûts de production à un faible niveau (recours limité aux intrants et à la complémentation). Or, dans de tels systèmes les ressources alimentaires pâturées (disponibilité et qualité) sont caractérisées par leur grande variabilité spatiale et temporelle et leur sensibilité aux aléas climatiques. Le milieu d'élevage étant faiblement contrôlé par l'éleveur, c'est sur l'animal et sur ses capacités adaptatives que reposent en grande partie la pérennité du troupeau et sa productivité. En particulier, la collecte de la ressource alimentaire étant principalement confiée à l'animal, celui-ci doit être capable d'assurer au mieux sa ration quotidienne par le pâturage et doit être capable de subir des périodes de sous-alimentation plus ou moins longues et intenses.

Dans cette synthèse, nous montrons que pour faire face à de telles contraintes de l'environnement, les herbivores sont capables de développer des réponses adaptatives qui mettent en œuvre des ajustements physiologiques et comportementaux. Ces réponses, lorsqu'elles sont analysées sur le long terme, révèlent des stratégies d'adaptation différentes entre races. Ces stratégies sont analysées du point de vue de leur efficacité et de leur aptitude à soutenir des priorités orientées vers la survie et le développement de l'organisme ou privilégiant l'investissement reproductif et maternel.

\begin{abstract}
Extensive ruminant farming systems highlight animal coping processes based on robustness, flexibility and plasticity

Extensive livestock systems are characterised by limited input. In such systems animal food requirements are mainly based on grazing. Vegetation varies in terms of space and time, and is heterogeneous and sensitive to climatic changes. The low control by the breeders on the immediate environment implies that the management of extensive systems in such a context mainly depends on the adaptive ability of the animals. They have to be able to fulfill their food requirements by developing specific behaviours such as land exploration and selective intake. They also have to cope with low feed intake periods of various intensities and duration.
\end{abstract}

In this paper we demonstrate that herbivores adjust their behaviour and physiological responses to cope with their environment and face constraints. Such adjustments highlight the differences between breeds in their ability to survive, produce and maintain their reproduction in a harsh unstable environment.

BLANC F., DUMONT B., BRUNSCHWIG G., BOCQUIER F., AGABRIEL J., 2010. Robustesse, flexibilité, plasticité : des processus adaptatifs révélés dans les systèmes d'élevage extensifs de ruminants. In : Robustesse, rusticité, flexibilité, plasticité, résilience... les nouveaux critères de qualité des animaux et des systèmes d'élevage. Sauvant D., Perez J.M. (Eds). Dossier Inra Prod. Anim., 23, 65-80. 